\title{
Reduced Heat Kernels on Nilpotent Lie Groups
}

\section{A.F.M. ter Elst ${ }^{1}$, Derek W. Robinson}

Centre for Mathematics and its Applications, School of Mathematical Sciences, Australian National University, Canberra, ACT 0200, Australia

Received: 19 September 1994

Abstract: Let $U$ be a basis representation of an irreducible unitary representation of a nilpotent Lie group $G$ in $L_{2}\left(\mathbf{R}^{k}\right)$ and let $d U$ denote the representation of the Lie algebra $\mathfrak{g}$ obtained by differentiation. If $b_{1}, \ldots, b_{d}$ is a basis of $\mathfrak{g}$ and $B_{l}=d U\left(b_{i}\right)$ we consider the operators

$$
H=-\sum_{l, j=1}^{d} c_{i j} B_{i} B_{j}+\sum_{i=1}^{d} c_{i} B_{i},
$$

where $C=\left(c_{l j}\right)$ is a real symmetric strictly positive matrix and $c_{i} \in \mathbf{C}$. Then $H$ generates a continuous semigroup $S$, holomorphic in the open right half-plane, with a reduced kernel $\kappa$ defined by

$$
\left(S_{z} \varphi\right)(x)=\int_{\mathbf{R}^{k}} d y \kappa_{z}(x ; y) \varphi(y) .
$$

We prove Gaussian off-diagonal bounds and "exponential" on-diagonal bounds for $\kappa$. For example, if $c_{l}=0$ we establish that

$$
\left|\kappa_{t}(x ; y)\right| \leqq a(1 \wedge \varepsilon \mu t)^{-k / 2} e^{-\lambda_{1} t} e^{-d(x ; y)^{2}(4(1+\varepsilon) t)^{-1}}
$$

for all $t>0$ and $\varepsilon \in\langle 0,1]$, where $\mu$ is the smallest eigenvalue of $C, \lambda_{1}$ is the smallest eigenvalue of $H$ and $d$ is a natural distance associated with the coefficients $C$ and the representation $U$. Bounds are also obtained for $c_{i} \neq 0$ and complex $t$. Alternatively, if $H$ is self-adjoint then

$$
\left|\kappa_{z}(x ; y)\right| \leqq a e^{-\lambda_{1} \operatorname{Re} z} e^{-b\left(|x|^{\alpha}+|y|^{\alpha}\right)}
$$

for all $z \in \mathbf{C}$ with $\operatorname{Re} z \geqq 1$, for some $\alpha \in\langle 0,2]$.

\footnotetext{
${ }^{1}$ Permanent address: Department of Mathematics and Computing Science, Eindhoven University of Technology, P.O. Box 513, 5600 MB Eindhoven, The Netherlands
} 


\section{Introduction}

The theory of strongly elliptic and subelliptic operators extends naturally from the Euclidean space $\mathbf{R}^{d}$ to a general Lie group $G$ (see, for example, [Rob, VSC]). In particular every strongly elliptic operator has a representative affiliated with each continuous Banach space representation $U$ of the group. This representative is a closable operator whose closure generates a continuous, holomorphic, semigroup $S$ with an action determined by an integral kernel $K$,

$$
S_{t}=\int_{G} d g K_{t}(g) U(g),
$$

where $d g$ denotes left-invariant Haar measure. The kernel $K$ is a universal, representation-independent, function whose smoothness and boundedness properties have been examined in detail. The kernel satisfies Gaussian upper bounds and for second-order operators with real coefficients it is positive and satisfies complementary Gaussian lower bounds. The derivation of good asymptotic estimates is, however, a more difficult and more specialized problem. The most detailed results have been derived for Laplacians and sublaplacians on unimodular Lie groups whose volume grows polynomially. In particular this includes all the nilpotent Lie groups. But in this latter context there are many new, interesting, representation-dependent, questions concerning the kernel.

The irreducible unitary representations of a $d$-dimensional, connected, simply connected, nilpotent Lie group $G$ are described by Kirillov theory [Kir]. If $l \in \mathfrak{g}^{*}$, the dual of the Lie algebra $\mathfrak{g}$ of $G$, and if $\mathrm{m} \subseteq \mathfrak{g}$ is a polarizing subalgebra of $l$ then $\chi(\exp a)=\exp (2 \pi i l(a))$ defines a one-dimensional representation of $M=\exp m$ from which one can induce a unitary representation of $G$ (see, for example, $[\mathrm{CoG}]$ ). Moreover, there is a one-to-one correspondence between the orbits in $\mathrm{g}^{*}$ under the coadjoint action of the group and the unitary dual of $G$. The induced representations corresponding to the pair $l$ and $m$ can be explicitly constructed on the space $L_{2}\left(\mathbf{R}^{k}\right)$, where $k$ is the codimension of $m$ in $\mathfrak{g}$, and other elements of $\mathfrak{g}^{*}$ on the orbit of $l$ and other polarizing subalgebras of $l$ induce unitarily equivalent representations of the group on $L_{2}\left(\mathbf{R}^{k}\right)$. We assume throughout that $k \geqq 1$ since the one-dimensional representations corresponding to the case $k=0$ offer no problem.

Now if $S$ is the semigroup generated by the closure of a strongly elliptic or subelliptic operator in a unitary representation corresponding to $l$ and $m$ then the action of $S$ is given by an integral kernel $\kappa$ on $\mathbf{R}^{k} \times \mathbf{R}^{k}$,

$$
\left(S_{t} \varphi\right)(x)=\int_{\mathbf{R}^{k}} d y \kappa_{t}(x ; y) \varphi(y)
$$

for all $\varphi \in L_{2}\left(\mathbf{R}^{k}\right)$. We refer to $\kappa$ as the reduced kernel. It is the central object of study in the sequel. The description reduced kernel is used because $\kappa$ is obtained from the universal kernel $K$ by first identifying it with a function over $\mathbf{R}^{d} \times \mathbf{R}^{d}$ by use of the exponential map and then "integrating out" the surplus variables (see [CoG] pp. 134-135). A key feature of this reduction process is that $K$ is multiplied by a complex-valued function prior to the integration. Therefore the reality and positivity properties of $K$ and $\kappa$ can be quite distinct. As an illustration let us consider the connected simply connected three-dimensional Heisenberg group.

Let $a_{1}, a_{2}, a_{3}$ be a basis of the Lie algebra $g$ of the Heisenberg group $G$ satisfying $\left[a_{1}, a_{2}\right]=a_{3}$ with the other commutators zero. Then the standard irreducible representation $U$ of $G$ on $L_{2}(\mathbf{R})$ is determined by exponentiation of 
the representation $d U\left(a_{1}\right)=-i P, d U\left(a_{2}\right)=i Q, d U\left(a_{3}\right)=i I$ of the Lie algebra $g$, where $(P f)(x)=i f^{\prime}(x)$ and $(Q f)(x)=x f(x)$ for all $f \in C_{c}^{\infty}(\mathbf{R})$ and $x \in \mathbf{R}$. The Laplacian corresponding to the standard basis $a_{1}, a_{2}, a_{3}$ is represented by

$$
H=-\sum_{i=1}^{3} d U\left(a_{i}\right)^{2}=P^{2}+Q^{2}+I=-\frac{d^{2}}{d x^{2}}+x^{2}+I .
$$

It is a positive self-adjoint operator and in addition is real, i.e., it leaves the real subspace of $L_{2}(\mathbf{R})$ invariant. If, however, one considers the Laplacians corresponding to the one-parameter family of bases $b_{1}=a_{1}+v a_{2}, b_{2}=a_{2}, b_{3}=a_{3}$, with $v \in \mathbf{R}$, then

$$
H_{v}=-\sum_{l=1}^{3} d U\left(b_{l}\right)^{2}=(P-v Q)^{2}+Q^{2}+I=-\left(\frac{d}{d x}+i v x\right)^{2}+x^{2}+I
$$

and $H_{v}, v \neq 0$, is not real although it is still positive and $H_{0}=H$. In fact one has

$$
H_{v}=e^{-i v Q^{2} / 2} H e^{i v Q^{2} / 2} .
$$

Now the reduced kernel $\kappa$ corresponding to $H$ is pointwise positive and is given by Mehler's formula;

$$
\kappa_{t}(x ; y)=\left(\pi\left(1-e^{-4 t}\right)\right)^{-1 / 2} e^{-(x+y)^{2}(\tanh t) / 4} e^{-(x-y)^{2}(\operatorname{coth} t) / 4} e^{-2 t}
$$

for all $t>0$ and $x, y \in \mathbf{R}$ (see [Dav 1] Theorem 7.13). But then the kernel $\kappa^{\prime \prime}$ corresponding to $H_{v}$ is given by

$$
\kappa_{t}^{v}(x ; y)=e^{-l v\left(x^{2}-y^{2}\right) / 2} \kappa_{t}(x ; y),
$$

and for $v \neq 0$ this is complex-valued. This is somewhat surprising as the $H_{v}$ are all Laplacians, albeit defined with different bases, and hence the corresponding universal kernels $K^{v}$ are strictly positive and satisfy Gaussian lower bounds (see, for example, [Rob] Sect. III.5). These observations clearly indicate that the analysis of the reduced kernels is quite different from that of the universal kernels.

The Heisenberg group also indicates the possible asymptotic properties of reduced kernels. For example,

$$
\left|\kappa_{t}^{v}(x ; x)\right| \sim(4 \pi t)^{-1 / 2} e^{-t x^{2}}
$$

for all small $t>0$ but

$$
\left|\kappa_{t}^{v}(x ; x)\right| \sim \pi^{-1 / 2} e^{-x^{2}} e^{-2 t}
$$

for large $t$. Thus the kernel is fast decreasing on the diagonal and for large $t$ the decrease is of the form $\exp \left(-\lambda_{1} t\right)$, where $\lambda_{1}=2$ is the smallest eigenvalue of $H_{v}$. Alternatively,

$$
\left|\kappa_{t}^{\prime \prime}(x+y / 2 ; x-y / 2)\right| \sim(4 \pi t)^{-1 / 2} e^{-y^{2} /(4 t)} e^{-t x^{2}}
$$

for all small $t>0$ but

$$
\left|\kappa_{t}^{v}(x+y / 2 ; x-y / 2)\right| \sim \pi^{-1 / 2} e^{-y^{2} / 4} e^{-2 t} e^{-x^{2}}
$$

for large $t$. Note that the Gaussian which dictates the off-diagonal decay for small $t$ has an exponent $1 / 4$ which is identical to that of the universal kernel (see [KuS]). 
Our aim is to establish broadly similar asymptotic estimates for reduced kernels for a general nilpotent group. The most precise results are for pure secondorder strongly elliptic operators with real symmetric coefficients but we also obtain estimates for more general second-order operators and higher-order operators with complex coefficients. There are two types of result which follow from two different approaches.

The first approach concentrates on the small $t$ behaviour and the off-diagonal decay of the reduced kernel. It consists of extending the Nash inequality methods of [Rob] and this involves tailoring the Nash inequalities to particular unitary representations. This enables us to establish that the kernels of $m^{\text {th }}$ order strongly elliptic operators have the expected singularity $t^{-k / m}$ for small $t>0$. Moreover, in the case of second-order operators $H$ with real principal coefficients one obtains Gaussian bounds $a_{\varepsilon} t^{-k / 2} \exp \left(-d(x ; y)^{2}(4(1+\varepsilon) t)^{-1}\right)$ for all $\varepsilon, t \in\langle 0,1]$. (The distance $d$ appearing in the estimates is the natural distance in $\mathbf{R}^{k}$ determined by the operator $H$ in the particular representation.) If the operator also has real first-order coefficients these estimates can be extended to all $t>0$ and one has an additional factor $\exp \left(-\lambda_{1} t\right)$, where $\lambda_{1}$ is the smallest eigenvalue of $H$. Thus one obtains bounds which closely approximate the optimal off-diagonal decay and incorporate the optimal large $t$ behaviour. Nevertheless, this approach gives no information about the on-diagonal decrease properties of the kernel.

The second approach concentrates on the large $t$ behaviour and the on-diagonal properties. It consists of a blend of spectral theory and Sobolev inequalities and applies to self-adjoint strongly elliptic or subelliptic operators of all orders. One derives bounds on the reduced kernel with the optimal decay $\exp \left(-\lambda_{1} t\right)$ for large $t$ which are "exponentially" decreasing along the diagonal. Estimates of this type have been previously obtained for Markov semigroups (see, for example, [Dav 2], Chapter 4) but the proofs depend heavily upon positivity arguments and hence are not applicable in the current context.

\section{Preliminaries}

As a preliminary to the estimation of semigroup kernels we first recall some further elements of Kirillov's theory of unitary representations and derive some useful results on particular representations and equivalences. Secondly, we give a precise definition of the reduced kernels and derive some of their simplest properties. Thirdly, we recall the definition of strongly elliptic operators and the associated semigroup kernels. For the Kirillov theory we mostly adopt the notation and terminology of Corwin and Greenleaf [CoG].

Let $G$ be a connected, simply connected, $d$-dimensional, nilpotent Lie group with Lie algebra $g$ and fix $l \in \mathfrak{g}^{*}$. Let $\mathrm{m}$ denote a polarizing subalgebra for $l$ of dimension $d_{\mathfrak{m}}$ and let $M=\exp (\mathrm{m})$ denote the corresponding subgroup of $G$. Further let $a_{1}, \ldots, a_{d_{\mathrm{m}}}, \ldots, a_{d_{\mathrm{m}}+k}$ be a weak Malcev basis of $\mathfrak{g}$ passing through $\mathrm{m}$, i.e., $\operatorname{span}\left\{a_{1}, \ldots, a_{j}\right\}$ is a subalgebra of $\mathfrak{g}$ for all $j \leqq d=d_{\mathfrak{m}}+k$ and $\mathrm{m}=$ $\operatorname{span}\left\{a_{1}, \ldots, a_{d_{m}}\right\}$. One can then define a one-dimensional representation of the subgroup $M$ by setting $\chi(\exp a)=\exp (2 \pi i l(a))$ for each $a \in \mathfrak{m}$ and this representation induces an irreducible unitary representation $\pi=\operatorname{ind}(M \uparrow G, \chi)$ on the Hilbert space $\mathscr{H}_{\pi}$ (see [CoG], Chapter 2). Explicitly, introduce a map $\gamma: \mathbf{R}^{k} \rightarrow G$ by

$$
\gamma(x)=\gamma\left(x_{1}, \ldots, x_{k}\right)=\exp \left(x_{1} a_{d_{\mathrm{u}}+1}\right) \cdots \exp \left(x_{k} a_{d_{\mathrm{m}}+k}\right) .
$$


The homogeneous space $M \backslash G$ of right cosets of the subgroup $M$ has a unique, up to a positive constant, right invariant measure $d \dot{g}$ given by the image of the Lebesgue measure of $\mathbf{R}^{k}$ under the analytic diffeomorphism $x \mapsto M \gamma(x)$. Next, let $\mathscr{H}_{\pi}$ be the Hilbert space of (equivalence classes) of Borel measurable functions $\varphi: G \rightarrow \mathbf{C}$ such that

$$
\varphi(m g)=\chi(m) \varphi(g)
$$

for all $m \in M$ and $g \in G$ and

$$
\int_{M \backslash G} d \dot{g}|\varphi(g)|^{2}<\infty .
$$

Then $(\pi(g) \varphi)(h)=\varphi(h g)$ defines a unitary representation of $G$ in $\mathscr{H}_{\pi}$, which is irreducible.

The map $(m, x) \mapsto m \cdot \gamma(x)$ is a diffeomorphism from $M \times \mathbf{R}^{k}$ onto $G$ and allows one to define a unitary map $J: L_{2}\left(\mathbf{R}^{k}\right) \rightarrow \mathscr{H}_{\pi}$ by

$$
(J \varphi)(m \gamma(x))=\chi(m) \varphi(x)
$$

for all $m \in M$ and $x \in \mathbf{R}^{k}$. One can then transfer the action $\pi$ of $G$ on $\mathscr{H}_{\pi}$ to a unitary action $U$ on $L_{2}\left(\mathbf{R}^{k}\right)$ by use of $J$. This is the basis realization of $\pi$ in [CoG], p. 125. The resulting representation depends on the choice of Malcev basis but each choice leads to a unitarily equivalent representation. An explicit description of the representation $U$ is as follows. Let $E=\left(E_{1}, E_{2}\right): G \rightarrow M \times \mathbf{R}^{k}$ be the inverse of the map $(m, x) \mapsto m \cdot \gamma(x)$. Then

$$
(U(g) \varphi)(x)=\chi\left(E_{1}(\gamma(x) g)\right) \varphi\left(E_{2}(\gamma(x) g)\right)
$$

for all $g \in G, \varphi \in L_{2}\left(\mathbf{R}^{k}\right)$ and almost all $x \in \mathbf{R}^{k}$. Moreover, $E_{1}$ and $E_{2}$ are polynomial maps. Note that $U$ depends on the weak Malcev basis only through $\operatorname{span}\left\{a_{1}, \ldots, a_{d_{\mathrm{m}}}\right\}$ and $a_{d_{\mathrm{m}}+1}, \ldots, a_{d_{\mathrm{m}}+k}$.

We begin by observing that the basis realization gives a simple result for the action of the representation on the $L_{p}$-spaces associated with the representation space.

Lemma 2.1. Let $U$ be a basis realization on $L_{2}\left(\mathbf{R}^{k}\right)$ of the induced representation $\pi$. Then $U$ extends to a continuous isometric representation on each of the spaces $L_{p}\left(\mathbf{R}^{k}\right), p \in[1, \infty]$.

Proof. For each $g \in G$ there is a polynomial $\sigma_{g}: \mathbf{R}^{k} \rightarrow \mathbf{R}$ and a polynomial diffeomorphism $\theta_{g}: \mathbf{R}^{k} \rightarrow \mathbf{R}^{k}$ such that

$$
(U(g) \varphi)(x)=e^{l \sigma_{g}(x)} \varphi\left(\theta_{g}(x)\right)
$$

for all $\varphi \in L_{2}\left(\mathbf{R}^{k}\right)$. This is just a restatement of (1). It is important that the Jacobian of the transformation $\theta_{g}$ has modulus one, since $U$ is unitary. Therefore

$$
\|U(g) \varphi\|_{1}=\int_{\mathbf{R}^{k}} d x\left|\varphi\left(\theta_{g}(x)\right)\right|=\int_{\mathbf{R}^{k}} d x|\varphi(x)|=\|\varphi\|_{1}
$$

for all $\varphi \in L_{1}\left(\mathbf{R}^{k}\right) \cap L_{2}\left(\mathbf{R}^{k}\right)$. Similarly, $\|U(g) \varphi\|_{\infty}=\|\varphi\|_{\infty}$ for all $\varphi \in L_{2}\left(\mathbf{R}^{k}\right) \cap$ $L_{\infty}\left(\mathbf{R}^{k}\right)$. Hence $U$ extends to a group of isometries on each of the $L_{p}$-spaces. Now 
continuity follows for $\varphi \in C_{c}^{\infty}(G)$ because

$$
\begin{aligned}
\|U(g) \varphi-\varphi\|_{p} \leqq & \left(\int_{\mathbf{R}^{k}} d x\left|\varphi\left(\theta_{g}(x)\right)-\varphi(x)\right|^{p}\right)^{1 / p} \\
& +\left(\int_{\mathbf{R}^{k}} d x\left|e^{l \sigma_{g}(x)}-1\right|^{p}|\varphi(x)|^{p}\right)^{1 / p} .
\end{aligned}
$$

The continuity is verified using the properties of $\sigma$ and $\theta$ together with the Lebesgue dominated convergence theorem. Strong continuity on $L_{p}\left(\mathbf{R}^{k}\right), p \in[1, \infty)$, follows by a density argument and weak* continuity on $L_{\infty}\left(\mathbf{R}^{k}\right)$ follows by duality.

In the subsequent proofs of kernel bounds some weak Malcev bases are more suitable than others in the basis realizations. We initially establish Nash inequalities for a basis realization of the representation associated with a weak Malcev basis with the following ideal property:

$$
\left[a, a_{d_{\mathrm{m}}+j}\right] \in \operatorname{span}\left\{a_{1}, \ldots, a_{d_{\mathfrak{m}}+j-1}\right\} \quad \text { for all } a \in \mathfrak{g} \text { and } j \in\{1, \ldots, k\} .
$$

These inequalities are then instrumental in the derivation of bounds on the reduced kernel in this particular realization of the unitary representation. Separate arguments are necessary to extend the bounds to other realizations.

Lemma 2.2. There exists a weak Malcev basis passing through the polarizing subalgebra $m$ with the ideal property (3).

Proof. One can easily construct a weak Malcev basis of in (see [CoG], Theorem 1.1.13(a)) and one has to extend this basis to a basis of $g$ with the property (3).

Therefore, given a proper subalgebra $\mathfrak{h}$ of $\mathfrak{g}$, one has to construct an element $a \in \mathfrak{g} \backslash \mathfrak{h}$ such that $[\mathfrak{g}, a] \subseteq \mathfrak{h}$. Then $\mathfrak{h}_{1}=\operatorname{span}(\mathfrak{h}, a)$ is a subalgebra of $\mathfrak{g}$ with $\operatorname{dim} \mathfrak{h}_{1}=1+\operatorname{dim} \mathfrak{h}$ and the lemma follows by induction. Let $\mathfrak{g}^{(n)}, n \in \mathbf{N}$, be the decreasing central series of $\mathfrak{g}$, i.e., $\mathfrak{g}^{(1)}=\mathfrak{g}$ and $\mathfrak{g}^{(n+1)}=\left[\mathfrak{g}, \mathfrak{g}^{(n)}\right]$. There exists $n \in \mathbf{N}$ such that $\mathfrak{g}^{(n+1)} \subseteq \mathfrak{h}$ but $\mathfrak{g}^{(n)} \nsubseteq \mathfrak{h}$. Let $a \in \mathfrak{g}^{(n)} \backslash \mathfrak{h}$. Then $[\mathfrak{g}, a] \subseteq\left[\mathfrak{g}, \mathfrak{g}^{(n)}\right]=$ $\mathfrak{g}^{(n+1)} \subseteq \mathfrak{h}$.

Thus for the given polarizing subalgebra $m$ one can always find a weak Malcev basis passing through $m$ which has the ideal property (3). We next examine the equivalence of two basis realizations corresponding to two weak Malcev bases passing through the same polarizing subalgebra.

Lemma 2.3. Let $a_{1}, \ldots, a_{d_{11}}, \ldots, a_{d}$ and $\tilde{a}_{1}, \ldots, \tilde{a}_{d_{\mathrm{m}}}, \ldots, \tilde{a}_{d}$ be two weak Malcev bases passing through $m$ and $U, \tilde{U}$, the corresponding basis realizations of the induced representation in $L_{2}\left(\mathbf{R}^{k}\right)$. Then there exist a polynomial $\sigma: \mathbf{R}^{k} \rightarrow \mathbf{R}, a$ polynomial diffeomorphism $\theta: \mathbf{R}^{k} \rightarrow \mathbf{R}^{k}$ and a constant $c>0$ such that the modulus of the Jacobian satisfies $\left|\operatorname{det} \theta^{\prime}(x)\right|=c^{2}$ for all $x \in \mathbf{R}^{k}$ and

$$
U=V \tilde{U} V^{*}
$$

where $V$ is the unitary map on $L_{2}\left(\mathbf{R}^{k}\right)$ defined by

$$
(V \varphi)(x)=c e^{i \sigma(x)} \varphi(\theta(x)) .
$$


Proof. Define the maps $\gamma: \mathbf{R}^{k} \rightarrow G, E_{1}: G \rightarrow M, E_{2}: G \rightarrow \mathbf{R}^{k}$ and $J: L_{2}\left(\mathbf{R}^{k}\right) \rightarrow \mathscr{H}_{\pi}$ as above with respect to the basis $a_{1}, \ldots, a_{d}$ and the analogous maps $\tilde{\gamma}, \tilde{E}_{1}$ and $\tilde{E}_{2}$ with respect to the basis $\tilde{a}_{1}, \ldots, \tilde{a}_{d}$. For the definition of $\tilde{J}$ one has to be careful since one can fix only once the measure on $M \backslash G$. This we did via the bijection $x \mapsto M \gamma(x)$. Therefore the image of Lebesgue measure under the map $x \mapsto M \tilde{\gamma}(x)$ equals a positive constant times the measure $d \dot{g}$ on $M \backslash G$. Hence there exists a $c>0$ such that

$$
(\tilde{J} \varphi)(m \tilde{\gamma}(x))=c \chi(m) \varphi(x)
$$

defines a unitary map from $L_{2}\left(\mathbf{R}^{k}\right)$ onto $\mathscr{H}_{\pi}$.

One now easily verifies that $V=J^{-1} \tilde{J}$ intertwines the representations $U$ and $\tilde{U}$ and $V$ is unitary. Moreover,

$$
(V \varphi)(x)=(\tilde{J} \varphi)(\gamma(x))=c \chi\left(\tilde{E}_{1} \gamma(x)\right) \varphi\left(\tilde{E}_{2} \gamma(x)\right)=c e^{i \sigma(x)} \varphi(\theta(x)),
$$

where $\theta=\tilde{E}_{2} \circ \gamma$ is a ploynomial from $\mathbf{R}^{k}$ into $\mathbf{R}^{k}$ and $\sigma(x)=2 \pi l\left(\exp ^{-1} \tilde{E}_{1} \gamma(x)\right)$ is a second ploynomial. It remains to show that $\theta$ is a polynomial diffeomorphism with a Jacobian whose modulus is equal to $c^{2}$.

Define $\tilde{\theta}: \mathbf{R}^{k} \rightarrow \mathbf{R}^{k}$ by $\tilde{\theta}=E_{2} \circ \tilde{\gamma}$. Then for all $x \in \mathbf{R}^{k}$ one has

$$
\theta(\tilde{\theta}(x))=\tilde{E}_{2} \gamma E_{2} \tilde{\gamma}(x)=\tilde{E}_{2}\left(\left(E_{1}(\tilde{\gamma}(x))\right)^{-1} \tilde{\gamma}(x)\right)=\tilde{E}_{2}(\tilde{\gamma}(x))=x
$$

and similarly $\tilde{\theta} \theta(x)=x$, so $\theta$ is a polynomial diffeomorphism. Then $x \rightarrow \operatorname{det} \theta^{\prime}(x)$ and $x \mapsto \operatorname{det} \tilde{\theta}^{\prime}(\theta(x))$ are polynomials and $\operatorname{det} \tilde{\theta}^{\prime}(\theta(x)) \cdot \operatorname{det} \theta^{\prime}(x)=\operatorname{det}(\tilde{\theta} \theta)^{\prime}(x)=1$. So $\operatorname{det} \theta^{\prime}$ is constant and non-zero. Since $V$ is unitary the absolute value of this constant must be equal to $c^{2}$.

Next we give a more precise definition of the reduced kernels. Let $\pi=\operatorname{ind}(M \uparrow$ $G, \chi)$ be the induced irreducible unitary representation on $\mathscr{H}_{\pi}$ described above. If $\tau \in \mathscr{P}(G)$ then the operator

$$
\pi(\tau)=\int_{G} d g \tau(g) \pi(g)
$$

is of trace class on $\mathscr{H}_{\pi}$ (see $[\mathrm{CoG}]$, Sect. 4.2). Moreover, in the basis realization $U$ of $\pi$ on $L_{2}\left(\mathbf{R}^{k}\right)$ corresponding to $l, m$ and a weak Malcev basis $a_{1}, \ldots, a_{d}$ passing through $\mathrm{nt}$, the action of $U(\tau)$ is determined by an integral kernel $\kappa_{\tau}$,

$$
(U(\tau) \varphi)(x)=\int_{\mathbf{R}^{k}} d y \kappa_{\tau}(x ; y) \varphi(y),
$$

where $\kappa_{\tau} \in \mathscr{S}\left(\mathbf{R}^{k} \times \mathbf{R}^{k}\right)$. Finally, $\kappa_{\tau}$ is given in terms of $\tau$ by the reduction formula

$$
\kappa_{\tau}(x ; y)=\int_{M} d m \chi(m) \tau\left(\gamma(x)^{-1} m \gamma(y)\right),
$$

where $\chi$ and $\gamma$ are the maps introduced earlier. This relation is of fundamental importance in the sequel.

There are some simple relationships between the kernels corresponding to unitarily equivalent representations. First we consider the relationship for kernels corresponding to different basis realizations.

Lemma 2.4. Let $U$ and $\tilde{U}$ be two basis realizations on $L_{2}\left(\mathbf{R}^{k}\right)$ of the induced representation $\pi$, as in Lemma 2.3, and $\kappa_{\tau}$ and $\tilde{\kappa}_{\tau}$ the kernels corresponding to 
the two representations and $\tau \in \mathscr{S}(G)$. Then

$$
\kappa_{\tau}(x ; y)=c^{2} e^{i(\sigma(x)-\sigma(y))} \tilde{\kappa}_{\tau}(\theta(x) ; \theta(y))
$$

for all $x, y \in \mathbf{R}^{k}$, where $\sigma, \theta, c$ are defined by Lemma 2.3 .

Proof. One has

$$
\begin{aligned}
\int_{\mathbf{R}^{k}} d x \int_{\mathbf{R}^{k}} d y \overline{\xi(x)} \kappa_{\tau}(x ; y) \psi(y) & =(\xi, U(\tau) \psi)=\left(V^{*} \xi, \tilde{U}(\tau) V^{*} \psi\right) \\
& =\int_{\mathbf{R}^{k}} d x \int_{\mathbf{R}^{k}} d y \overline{\left(V^{*} \xi\right)(x)} \tilde{\kappa}_{\tau}(x ; y)\left(V^{*} \psi\right)(y)
\end{aligned}
$$

for all $\xi, \psi \in L_{2}\left(\mathbf{R}^{k}\right)$, where

$$
\left(V^{*} \xi\right)(x)=c^{-1} e^{-i \sigma\left(\theta^{-1}(x)\right)} \xi\left(\theta^{-1}(x)\right) .
$$

Therefore, since $c^{2}$ is the absolute value of the Jacobian of the transformation $x \mapsto \theta(x)$ one immediately finds the desired relation between the two kernels.

Secondly, we compare the kernels corresponding to shifts under the group. If $\pi$ is a unitary representation of $G$ on $\mathscr{H}_{\pi}$ then for each $h \in G$ one has a unitarily equivalent representation $\pi_{h}$ given by $\pi_{h}(g)=\pi\left(h g h^{-1}\right)=\pi(h) \pi(g) \pi\left(h^{-1}\right)$. Moreover, if $\pi$ is the induced representation corresponding to $l$ and $m$ then $\pi_{h}$ is the induced representation corresponding to the images $l_{h}$ and $\mathrm{m}_{h}$ of $l$ and $\mathrm{m}$ under the coadjoint and adjoint action of the group, respectively. Furthermore, if $U$ denotes the basis realization of $\pi$ on $L_{2}\left(\mathbf{R}^{k}\right)$ corresponding to a weak Malcev basis passing through $\mathrm{m}$ then there is a realization $U_{h}$ corresponding to the images of $l, \mathrm{~m}$ and the basis. But for each $h \in G$ there is a polynomial $\sigma_{h}: \mathbf{R}^{k} \rightarrow \mathbf{R}$ and a polynomial diffeomorphism $\theta_{h}: \mathbf{R}^{k} \rightarrow \mathbf{R}^{k}$ such that

$$
(U(h) \varphi)(x)=e^{i \sigma_{h}(x)} \varphi\left(\theta_{h}(x)\right)
$$

for all $\varphi \in L_{2}\left(\mathbf{R}^{k}\right)$. This is again a rephrasing of (1) and again the Jacobian of the transformation $\theta_{h}$ has modulus one. Therefore, if $\kappa_{\tau}$ and $\kappa_{\tau}^{h}$ are the kernels corresponding to $U$ and $U_{h}$ and $\tau \in \mathscr{S}(G)$ then

$$
\kappa_{\tau}^{h}(x ; y)=e^{i\left(\sigma_{h}(x)-\sigma_{h}(y)\right)} \kappa_{\tau}\left(\theta_{h}(x) ; \theta_{h}(y)\right)
$$

for all $x, y \in \mathbf{R}^{k}$. This is the direct analogue of the conclusion of Lemma 2.4 for the kernels corresponding to representations arising from different Malcev bases passing through the same polarizing subalgebra. Nevertheless, unitary equivalence of representations does not always imply that the kernels are related in the manner of (5). There is a third form of unitary equivalence of induced representations for which the relationship between the kernels is quite different.

If $l \in \mathrm{g}^{*}$ and $\mathrm{m}_{1}, \mathrm{~m}_{2}$ are two different polarizing subalgebras then the induced representations $\pi_{1}$ and $\pi_{2}$ corresponding to $\left(l, m_{1}\right)$ and $\left(l, m_{2}\right)$ are unitarily equivalent. But the connection between the reduced kernels $\kappa_{\tau}^{(1)}$ and $\kappa_{\tau}^{(2)}$ associated with a $\tau \in \mathscr{S}(G)$ and two weak Malcev bases is not generally of the above form. For example, consider the case that $m_{1}$ and $m_{2}$ have codimension one in $g$ but $\mathfrak{m}_{1} \cap \mathrm{m}_{2}$ has codimension two. Then one can choose elements $a_{1}, \ldots, a_{d} \in \mathfrak{g}$ such that $a_{1}, \ldots, a_{d-2}, a_{d-1}, a_{d}$ is a weak Malcev basis passing 
through $\mathrm{m}_{1}, a_{1}, \ldots, a_{d-2}, a_{d}, a_{d-1}$ is a weak Malcev basis passing through $\mathrm{m}_{2}$, and $l\left(\left[a_{d-1}, a_{d}\right]\right)=1$. The corresponding unitarily equivalent representations $U_{1}$ and $U_{2}$ on $L_{2}(\mathbf{R})$ can then be expressed as

$$
\begin{aligned}
& d U_{1}\left(a_{d}\right)=\frac{\partial}{\partial x}+2 \pi i l\left(a_{d}\right), \quad d U_{1}\left(a_{d-1}\right)=-2 \pi i x+2 \pi i l\left(a_{d-1}\right), \\
& d U_{2}\left(a_{d}\right)=2 \pi i x+2 \pi i l\left(a_{d}\right), \quad d U_{2}\left(a_{d-1}\right)=\frac{\partial}{\partial x}+2 \pi i l\left(a_{d-1}\right),
\end{aligned}
$$

and

$$
d U_{1}(a)=d U_{2}(a)=2 \pi i l(a)
$$

for all $a \in \operatorname{span}\left\{a_{1}, \ldots, a_{d-2}\right\}$. Now, however, the unitary equivalence of the representations is given by Fourier transformation and the kernels are linked by the relation

$$
\kappa_{\tau}^{(1)}(x ; y)=\left(\mathscr{F} \kappa_{\tau}^{(2)}\right)(-x ; y)
$$

where $\mathscr{F}$ denotes the Fourier transform with respect to both variables.

Next we recall some basic properties of strongly elliptic operators on Lie groups and the corresponding semigroups. We mostly follow the notation and terminology of [Rob].

Each strongly elliptic operator on the $d$-dimensional Lie group $G$ is defined in terms of a basis $b_{1}, \ldots, b_{d}$ of the Lie algebra $\mathbf{g}$ and a form $C$, i.e., a family $c_{\alpha} \in \mathbf{C}$ of complex-valued coefficients indexed by a multi-index $\alpha=\left(\alpha_{1}, \ldots, \alpha_{d}\right)$ with $\alpha_{l} \in \mathbf{N}_{0}$ and $|\alpha|=\alpha_{1}+\cdots+\alpha_{d}$. The form $C$ is called an $m^{\text {th }}$ order strongly elliptic form if $c_{\alpha}=0$ for $|\alpha|>m$ and the ellipticity constant

$$
\mu_{C}=\inf \left\{\operatorname{Re} \sum_{\alpha:|\alpha|=m} c_{\alpha}(i \xi)^{\alpha}: \xi \in \mathbf{R}^{d},|\xi|=1\right\}
$$

is strictly positive. Given the basis and the strongly elliptic form one can define a strongly elliptic element of the complex universal enveloping algebra $\mathbb{6} \mathfrak{b} \mathrm{g}$ by

$$
h_{m}=\sum_{\alpha:|\alpha| \leqq m} c_{\alpha} b^{\alpha}
$$

where $b^{\alpha}=b_{1}^{\alpha_{1}} \cdots b_{d}^{\alpha_{d}}$. There is a unique anti-automorphism $a \mapsto a^{\dagger}$ on 65 such that $x^{\dagger}=-x$ for all $x \in \mathfrak{g}$ and the image $h_{m}^{\dagger}$ of $h_{m}$ under this mapping is called the formal adjoint of $h_{m}$. It is a strongly elliptic element,

$$
h_{m}^{\dagger}=\sum_{\alpha:|\alpha| \leqq m} c_{\alpha}^{\dagger} b^{\alpha}
$$

with coefficients $c_{\alpha}^{\dagger}$ uniquely determined by the $c_{\alpha}$ and with $c_{\alpha}^{\dagger}=\overline{c_{\alpha}}$ if $|\alpha|=m$.

Next let $(\mathscr{X}, U, G)$ be a continuous representation of $G$ on the Banach space $\mathscr{X}$ and let $B_{i}=d U\left(b_{l}\right)$ denote the generator of the one-parameter subgroup $t \mapsto U\left(\exp \left(-t b_{i}\right)\right)$. Then there is a densely defined, closable, operator $H_{m}$ on $\chi$ 
such that

$$
H_{m}=d U(C)=\sum_{\alpha:|\alpha| \leqq m} c_{\alpha} B^{\alpha}
$$

with $B^{\alpha}=B_{1}^{\alpha_{1}} \cdots B_{d}^{\alpha_{d}}$ and $D\left(H_{m}\right)$ is the common domain $\mathscr{X}_{m}$ of all the $B^{\alpha}$ with $|\alpha|=m$. The formal adjoint $H_{m}^{\dagger}$ of $H_{m}$ is defined in an analogous manner from $h_{m}^{\dagger}$. These operators are called $m^{\text {th }}$ order strongly elliptic operators and the coefficients $c_{\alpha}$ with $|\alpha|=m$ are called the principal coefficients.

Second-order operators can be reexpressed in the form

$$
H_{2}=-\sum_{l, j=1}^{d} c_{l j} A_{l} A_{J}+\sum_{i=1}^{d} c_{i} A_{i}+c_{0} I,
$$

where the matrix $C=\left(c_{l j}\right)$ of principal coefficients is strictly positive and symmetric. The ellipticity constant is then identified as the smallest eigenvalue of $C$. In the sequel we will consider second-order operators for which the principal coefficients $c_{i j}$ are real.

The basic results we need are the following.

The closure $\overline{H_{m}}$ of the strongly elliptic operator $H_{m}$ generates a continuous semigroup $S$ on $\mathscr{X}$ with a universal kernel $K_{t} \in \mathscr{S}(G)$ which depends only on the basis $b_{1}, \ldots, b_{d}$ and the form $C$, i.e., $S_{t}=U\left(K_{t}\right)$ with $K_{t}$ independent of the particular representation. The kernel satisfies Gaussian bounds of order $m$,

$$
\left|K_{t}(g)\right| \leqq a t^{-d / m} e^{\omega t} e^{-b\left(|g|^{m} t^{-1}\right)^{1 /(m-1)}},
$$

where $a, b>0, \omega \geqq 0$ and $g \mapsto|g|$ is a modulus on the group. The kernel is positive if and only if the operator is of second-order with real coefficients. Finally, the kernel $K^{\dagger}$ corresponding to the formal adjoint satisfies

$$
K_{t}^{\dagger}(g)=\Delta(g)^{-1} \overline{K_{t}\left(g^{-1}\right)}
$$

where $\Delta$ is the modular function on $G$.

In fact there exists $\theta \in\langle 0, \pi / 2]$ such that for any $g \in G$ the function $t \mapsto K_{t}(g)$ extends to a function which is holomorphic in the subsector $\{z \in \mathbf{C}:|\arg z|<\theta\}$ of the right half plane and $S_{t}=U\left(K_{t}\right)$ extends to a holomorphic semigroup on the sector $\{z \in \mathbf{C}:|\arg z|<\theta\}$. Note that this subsector is representation independent. Moreover, $\theta=\pi / 2$ if the principal coefficients are real. The Gaussian bounds extend to this universal subsector but the relation with the formal adjoint becomes

$$
K_{z}^{\dagger}(g)=\Delta(g)^{-1} \overline{K_{\bar{z}}\left(g^{-1}\right)} .
$$

If the Lie group $G$ is nilpotent then there are a number of properties of the semigroup generated by the strongly elliptic operator in the irreducible representations which follow from the general theory.

Let $U$ be a basis realization on $L_{2}\left(\mathbf{R}^{k}\right)$ of the induced representation $\pi$ of the nilpotent group and $K_{t}$ the kernel corresponding to the strongly elliptic element $h_{m}$ of $\left(5\right.$. Since $K_{t} \in \mathscr{S}(G)$ there is a reduced kernel $\kappa_{t} \in \mathscr{S}\left(\mathbf{R}^{k} \times \mathbf{R}^{k}\right)$ defined by the analogue of (4),

$$
\kappa_{t}(x ; y)=\int_{M} d m \chi(m) K_{t}\left(\gamma(x)^{-1} m \gamma(y)\right) .
$$


Then the semigroup $S$ corresponding to $h_{m}$ in the representation $U$ on $L_{2}\left(\mathbf{R}^{k}\right)$ is given by

$$
\left(S_{t} \varphi\right)(x)=\left(U\left(K_{t}\right) \varphi\right)(x)=\int_{\mathbf{R}^{k}} d y \kappa_{t}(x ; y) \varphi(y) .
$$

Note that as a consequence of Lemma 2.1 and the general theory of strongly elliptic operators the semigroup $S$ extends from $L_{2}\left(\mathbf{R}^{k}\right)$ to a continuous semigroup on each of the spaces $L_{p}\left(\mathbf{R}^{k}\right), p \in[1, \infty]$. Moreover,

$$
\left\|S_{t}\right\|_{1 \rightarrow 1} \leqq\left\|K_{t}\right\|_{1}, \quad\left\|S_{t}\right\|_{\infty \rightarrow \infty} \leqq\left\|K_{t}^{\dagger}\right\|_{1}=\left\|K_{t}\right\|_{1}
$$

and, by interpolation,

$$
\left\|S_{t}\right\|_{p \rightarrow p} \leqq\left\|K_{t}\right\|_{1}
$$

Since $K$ is universal these bounds are representation independent.

Similar properties are true for complex $t$ in the universal sector of holomorphy. The reduced kernel is defined by (6),

$$
\kappa_{z}(x ; y)=\int_{M} d m \chi(m) K_{z}\left(\gamma(x)^{-1} m \gamma(y)\right),
$$

and $z \mapsto \kappa_{z}(x ; y)$ remains holomorphic in the subsector. This follows from the Gaussian bounds on $K$ and the estimates of Lemma 4.2.3 in [CoG]. Combination of these estimates with the Gaussian bounds guarantees that the integral relating $K$ and $\kappa$ is convergent uniformly on compact subsets of $\mathbf{R}^{k} \times \mathbf{R}^{k}$. The action of $S_{z}$ is determined by $\kappa_{z}$ within the universal subsector of holomorphy as a consequence of the general theory. Now, however, one has

$$
\left\|S_{z}\right\|_{1 \rightarrow 1} \leqq\left\|K_{z}\right\|_{1}, \quad\left\|S_{z}\right\|_{\infty \rightarrow \infty} \leqq\left\|K_{z}^{\dagger}\right\|_{1}=\left\|K_{\bar{z}}\right\|_{1}
$$

and interpolation gives

$$
\left\|S_{z}\right\|_{p \rightarrow p} \leqq\left\|K_{z}\right\|_{1}^{1 / p}\left\|K_{\bar{z}}\right\|_{1}^{1-1 / p} .
$$

Again these bounds are representation independent.

We next establish that the $S_{t}, t>0$, are compact operators on the $L_{p}$-spaces and the semigroup generator has a compact resolvent on each of these spaces.

Theorem 2.5. Let $l \in \mathfrak{g}^{*}, a_{1}, \ldots, a_{d_{m}}, \ldots, a_{d_{m}+k}$ a weak Malcev basis passing through a polarizing subalgebra $\mathrm{m}$ of $l$ and $U$ the corresponding basis realization on $L_{2}\left(\mathbf{R}^{k}\right)$. Next, let $C$ be a strongly elliptic form of order $m, p \in[1, \infty]$ and $H_{m}=d U(C)$ the corresponding strongly elliptic operator on $L_{p}\left(\mathbf{R}^{k}\right)$. Then the spectrum of the closure of $H_{m}$ is a countable discrete set with accumulation point at infinity and each point in the spectrum corresponds to an eigenvalue of finite multiplicity. Moreover, the spectrum and the eigenspaces are independent of $p$.

Proof. If $t>0$ and $p, q \in[1, \infty]$, then $S_{t}$ is a continuous operator from $L_{p}\left(\mathbf{R}^{k}\right)$ into $L_{q}\left(\mathbf{R}^{k}\right)$ since $\kappa_{t} \in \mathscr{S}\left(\mathbf{R}^{k} \times \mathbf{R}^{k}\right)$. So for all $p \in[1, \infty]$ the operator $S_{t}=$ $S_{t / 3} \circ S_{t / 3} \circ S_{t / 3}: L_{p} \rightarrow L_{2} \rightarrow L_{2} \rightarrow L_{p}$ is compact since $S_{t / 3}=U\left(K_{t / 3}\right): L_{2} \rightarrow L_{2}$ is compact (see [CoG], Theorem 4.2.1). 
Next, if $p \in[1, \infty)$ and $\lambda>0$ is large enough then the integral

$$
\left(\lambda I+\overline{H_{m}}\right)^{-1}=\int_{0}^{\infty} d t e^{-\lambda t} S_{t}
$$

is norm convergent in $\mathscr{L}\left(L_{p}\right)$, so $\left(\lambda I+\overline{H_{m}}\right)^{-1}$ is compact from $L_{p}$ into $L_{p}$. By duality, the resolvent operator $\left(\lambda I+\overline{H_{m}}\right)^{-1}=\left(\left(\lambda I+\overline{H_{m}^{\dagger}}\right)^{-1}\right)^{*}$ is also compact from $L_{\infty}$ into $L_{\infty}$. The spectrum of $\overline{H_{m}}$ must have an accumulation point at infinity since the representation space is infinite dimensional.

Finally, let $\varphi \in L_{p}$ be an eigenvector for the operator $\overline{H_{m}}$ on $L_{p}$ with eigenvalue $\lambda$. Then $\varphi \in D^{\infty}\left(\overline{H_{m}}\right)=\mathscr{S}\left(\mathbf{R}^{k}\right)$, by [CoG] Theorem 4.1.1(i) and the Sobolev embedding theorem. Hence $\varphi \in L_{q}$, for all $q \in[1, \infty]$ and $H \varphi=\lambda \varphi$ in $L_{q}$. Thus the spectra and eigenspaces are independent of $p$.

In Sect. 5 we will derive some crude estimates on the growth behaviour of the eigenvalues in order to establish bounds on the reduced kernel for large time.

\section{Young and Nash Inequalities}

Our aim is to derive bounds on the reduced semigroup kernel $\kappa_{t}$ defined by (6) in an arbitrary irreducible unitary representation of the group. We accomplish this in two steps. First, we derive bounds with the correct singular structure for small values of $t$. Secondly, by a separate argument, we establish bounds with the correct asymptotic decrease for large $t$. The derivation of small $t$ bounds on the universal kernel $K$ in [Rob], Chapter IV, via Nash inequalities extends to give the small $t$ bounds, but this extension requires a form of the Nash inequalities tailored to the particular unitary representation. We begin by considering a particular basis realization of the representation.

Let $U$ be the basis realization of the nilpotent Lie group $G$ corresponding to a weak Malcev basis $a_{1}, \ldots, a_{d_{m}}, \ldots, a_{d_{m}+k}$ passing through a polarizing subalgebra $\mathrm{m}$ for an $l \in \mathfrak{g}^{*}$. If $\varphi \in L_{2}\left(\mathbf{R}^{k}\right)$ and $\psi \in L_{1}(G ; d g)$ one can define a convolution product $\psi *_{U} \varphi$ by introducing

$$
U(\psi)=\int_{G} d g \psi(g) U(g),
$$

and then setting

$$
\psi *_{U} \varphi=U(\psi) \varphi
$$

The aim of this section is to establish a version of Young's inequality for this product whenever the weak Malcev basis has the ideal property (3). Therefore we introduce the space $\mathscr{L}_{q}$ with $q \in[1, \infty]$ as the set of (equivalence classes of) measurable functions $\psi$ over $\mathbf{R}^{d_{m}} \times \mathbf{R}^{k}$ for which the norm \|\|$\psi\|\|_{q}$ is finite where

$$
\|\| \psi \|_{q}=\int_{\mathbf{R}^{d_{m}}} d w\left(\int_{\mathbf{R}^{k}} d x|\psi(\beta(w) \gamma(x))|^{q}\right)^{1 / q}
$$

for $q \in[1, \infty\rangle$,

$$
\left\|\left|\psi \|_{\infty}=\int_{\mathbf{R}^{d_{m}}} d w \operatorname{ess} \sup _{x \in \mathbf{R}^{k}}\right| \psi(\beta(w) \gamma(x)) \mid\right.
$$


and $\beta: \mathbf{R}^{d_{m}} \rightarrow M$ is the map

$$
\beta\left(w_{1}, \ldots, w_{d_{m}}\right)=\exp \left(w_{1} a_{1}\right) \cdots \exp \left(w_{d_{m}} a_{d_{m}}\right) .
$$

Note that the product $*_{U}$ and the spaces $\mathscr{L}_{q}, q \neq 1$ all depend on the choice of basis. Only the space $\mathscr{L}_{1}$ is independent of the basis since $\mathscr{L}_{1}=L_{1}(G)$.

Proposition 3.1. Let $a_{1}, \ldots, a_{d_{m}}, \ldots, a_{d_{m}+k}$ be a weak Malcev basis passing through $m$ which has the ideal property (3). If $p, q, r \in[1, \infty]$ and $1+1 / r=1 / p+1 / q$ then $\psi *_{U} \varphi \in L_{r}\left(\mathbf{R}^{k}\right)$ and

$$
\left\|\psi *_{U} \varphi\right\|_{r} \leqq\|\varphi\|_{p}\|\| \psi \|_{q}
$$

for all $\varphi \in L_{p}\left(\mathbf{R}^{k}\right) \cap L_{2}\left(\mathbf{R}^{k}\right)$ and $\psi \in \mathscr{L}_{q} \cap L_{1}(G)$. Hence the map $(\psi, \varphi) \mapsto \psi *_{U} \varphi$ from $\left(\mathscr{L}_{q} \cap \mathscr{L}_{1}\right) \times\left(L_{p}\left(\mathbf{R}^{k}\right) \cap L_{2}\left(\mathbf{R}^{k}\right)\right)$ into $L_{r}\left(\mathbf{R}^{k}\right)$ can be extended to a map from $\mathscr{L}_{q} \times L_{p}\left(\mathbf{R}^{k}\right)$ into $L_{r}\left(\mathbf{R}^{k}\right)$, which we will still denote by $*_{U}$, and

$$
\left\|\psi *_{U} \varphi\right\|_{r} \leqq\|\varphi\|_{p}\|\| \psi \|_{q}
$$

for all $\varphi \in L_{p}\left(\mathbf{R}^{k}\right)$ and $\psi \in \mathscr{L}_{q}$.

Remark 3.2. The inequalities of the proposition correspond to the classical Young inequalities when $G=\mathbf{R}^{k}$ and $U$ is the action by translation.

The proof of the proposition relies on a combinatorial result for products of exponentials, an interpolation property of the spaces $\mathscr{L}_{q}$ and adaptation of the interpolation proof of the classical Young inequalities.

Lemma 3.3. Let $a_{1}, \ldots, a_{d_{m}}, \ldots, a_{d_{m}+k}$ be a weak Malcev basis passing through $\mathrm{m}$ which has the ideal property (3). If $w \in \mathbf{R}^{d_{m}}$ and $x, y \in \mathbf{R}^{k}$ then there exist an $m\left(=m_{w, x, y}\right) \in M$ and $a z\left(=z_{w, x, y}\right) \in \mathbf{R}^{k}$ such that

$$
\gamma(x) \beta(w) \gamma(y)=m \gamma(z) .
$$

Moreover, there exist polynomials $p_{1}, \ldots, p_{k-1}$ such that

$$
\begin{aligned}
z_{k} & =y_{k}+x_{k}, \\
z_{k-1} & =y_{k-1}+x_{k-1}+p_{k-1}\left(w, y_{k}, x_{k}\right), \\
z_{k-2} & =y_{k-2}+x_{k-2}+p_{k-2}\left(w, y_{k}, x_{k}, y_{k-1}, x_{k-1}\right), \\
& \vdots \\
z_{1} & =y_{1}+x_{1}+p_{1}\left(w, y_{k}, x_{k}, \ldots, y_{2}, x_{2}\right),
\end{aligned}
$$

where the $p$, only depend on the indicated variables.

Proof. By using the Baker-Campbell-Hausdorff formula one can reexpress the product $\gamma(x) \beta(w) \gamma(y)$ as a single exponential and then separate the terms starting with $z_{k}, z_{k-1}, \ldots$. It follows from this process and the ideal property (3) of the Malcev basis that the product can be expressed in the desired form.

The most important implication of the lemma for the subsequent calculations is summarized in the following corollary. 
Corollary 3.4. If $z_{w, x, y}$ is defined by Lemma 3.3, then the maps $x \mapsto z_{w, x, y}$ and $y \mapsto z_{w, x, y}$ from $\mathbf{R}^{k}$ into $\mathbf{R}^{k}$ are bijections and have Jacobian one.

Proof. The Jacobi matrices are triangular and the diagonal elements are all equal to one. Therefore the determinants have value one.

Lemma 3.5. Let $p_{1}, p_{2}, q_{1}, q_{2} \in[1, \infty]$. If $T$ is a linear operator from the space $\mathscr{L}_{p_{1}} \cap \mathscr{L}_{p_{2}}$ to the space $L_{q_{1}}\left(\mathbf{R}^{k}\right) \cap L_{q_{2}}\left(\mathbf{R}^{k}\right)$ and

$$
\|T \psi\|_{q_{1}} \leqq M_{1}\|\| \psi\|\|_{p_{1}}, \quad\|T \psi\|_{q_{2}} \leqq M_{2}\|\| \psi\|\|_{p_{2}}
$$

then $T$ extends to a bounded linear operator from $\mathscr{L}_{p_{\gamma}}$ to $L_{q_{\gamma}}\left(\mathbf{R}^{k}\right)$ with norm less than or equal to $M_{1}^{1-\gamma} M_{2}^{\gamma}$, where

$$
p_{\gamma}^{-1}=(1-\gamma) p_{1}^{-1}+\gamma p_{2}^{-1}, \quad q_{\gamma}^{-1}=(1-\gamma) q_{1}^{-1}+\gamma q_{2}^{-1}
$$

and $\gamma \in[0,1]$.

This is just a variant of the Riesz-Thorin interpolation theorem which is established by a slight modification of the arguments used to prove the classical version.

Now we are prepared to prove the proposition.

Proof of Proposition 3.1. First, consider the case $p=q=r=1$. Let $\varphi \in L_{1}\left(\mathbf{R}^{k}\right) \cap$ $L_{2}\left(\mathbf{R}^{k}\right)$ and $\psi \in L_{1}(G)$. Since $U$ extends to an isometric continuous representation on $L_{1}\left(\mathbf{R}^{k}\right)$ one has

$$
\left\|\psi *_{U} \varphi\right\|_{1}=\|U(\psi) \varphi\|_{1} \leqq\|\psi\|_{1}\|\varphi\|_{1}
$$

Since $\mathscr{L}_{1}=L_{1}(G)$ and \|\|$\psi\left\|_{1}=\right\| \psi \|_{1}$ this establishes the special case of the desired result.

Secondly, we consider the case $p=1$ and $q=r=\infty$. Let $\varphi \in L_{1}\left(\mathbf{R}^{k}\right) \cap L_{2}\left(\mathbf{R}^{k}\right)$ and $\psi \in \mathscr{L}_{1} \cap \mathscr{L}_{\infty}$. Then it follows from (1) that

$$
\begin{aligned}
(U(\psi) \varphi)(x) & =\int_{\mathbf{R}^{d_{m}}} d w \int_{\mathbf{R}^{k}} d y \psi(\beta(w) \gamma(y))(U(\beta(w) \gamma(y)) \varphi)(x) \\
& =\int_{\mathbf{R}^{d_{m}}} d w \int_{\mathbf{R}^{k}} d y \psi(\beta(w) \gamma(y)) \chi\left(m_{w, x, y}\right) \varphi\left(z_{w, x, y}\right)
\end{aligned}
$$

where we have used the notation of Lemma 3.3 in the last step. Therefore

$$
\left(\psi *_{U} \varphi\right)(x)=\int_{\mathbf{R}^{d_{m}}} d w \int_{\mathbf{R}^{k}} d y \psi(\beta(w) \gamma(y)) \chi\left(m_{w, x, y}\right) \varphi\left(z_{w, x, y}\right)
$$

and hence

$$
\left|\left(\psi *_{U} \varphi\right)(x)\right| \leqq \int_{\mathbf{R}^{d_{m}}} d w \int_{\mathbf{R}^{k}} d y|\psi(\beta(w) \gamma(y))| \cdot\left|\varphi\left(z_{w, x, y}\right)\right|
$$


Therefore

$$
\begin{aligned}
\left\|\psi *_{U} \varphi\right\|_{\infty} & \leqq \sup _{x \in \mathbf{R}^{k} \mathbf{R}^{d_{m}}} d w \int_{\mathbf{R}^{k}} d y|\psi(\beta(w) \gamma(y))| \cdot\left|\varphi\left(z_{w, x, y}\right)\right| \\
& \leqq \sup _{x \in \mathbf{R}^{k} \mathbf{R}^{d_{m}}} d w \int_{\mathbf{R}^{k}} d y \sup _{y^{\prime} \in \mathbf{R}^{k}}\left|\psi\left(\beta(w) \gamma\left(y^{\prime}\right)\right)\right| \cdot\left|\varphi\left(z_{w, x, y}\right)\right| \\
& =\int_{\mathbf{R}^{d_{m}}} d w \int_{\mathbf{R}^{k}} d z \sup _{y^{\prime} \in \mathbf{R}^{k}}\left|\psi\left(\beta(w) \gamma\left(y^{\prime}\right)\right)\right| \cdot|\varphi(z)| \\
& =\|\| \psi\left\|_{\infty}\right\| \varphi \|_{1},
\end{aligned}
$$

where the third step uses a change of variables $y \mapsto z_{w, x, y}$ and Corollary 3.4.

Thirdly, we interpolate between the estimates (7) and (9).

The estimate (7) states that for $\varphi \in L_{1}\left(\mathbf{R}^{k}\right) \cap L_{2}\left(\mathbf{R}^{k}\right)$ the linear operator $T_{\varphi}$ defined by

$$
T_{\varphi} \psi=\psi *_{U} \varphi
$$

is bounded from $\mathscr{L}_{1}$ to $L_{1}\left(\mathbf{R}^{k}\right)$ and

$$
\left\|T_{\varphi} \psi\right\|_{1} \leqq\|\varphi\|_{1}\|\psi \psi\|_{1} .
$$

Similarly, (9) states that the operator $T_{\varphi}$ is bounded from $\mathscr{L}_{1} \cap \mathscr{L}_{\infty}$ to $L_{\infty}\left(\mathbf{R}^{k}\right)$ and

$$
\left\|T_{\varphi} \psi\right\|_{\infty} \leqq\|\varphi\|_{1}\|\psi \psi\|_{\infty}
$$

Therefore it follows from Lemma 3.5 that $T_{\varphi}$ extends to a bounded operator from $\mathscr{L}_{q}$ to $L_{q}\left(\mathbf{R}^{k}\right)$ for each $q \in[1, \infty]$ and

$$
\left\|\psi *_{U} \varphi\right\|_{q}=\left\|T_{\varphi} \psi\right\|_{q} \leqq\|\varphi\|_{1}\|\psi \psi\| \|_{q}
$$

Fourthly, the Hölder inequality gives

$$
\begin{aligned}
\left\|\psi *_{U} \varphi\right\|_{\infty} & \leqq \sup _{x \in \mathbf{R}^{k} \mathbf{R}^{d_{m}} \int_{\mathbf{R}^{k}} d w \int_{\mathbf{R}^{k}} d y|\psi(\beta(w) \gamma(y))| \cdot\left|\varphi\left(z_{w, x, y}\right)\right|} \\
& \leqq \sup _{x \in \mathbf{R}^{k}} \int_{\mathbf{R}^{d_{m}}} d w\left(\int_{\mathbf{R}^{k}} d y|\psi(\beta(w) \gamma(y))|^{q}\right)^{1 / q}\left(\int_{\mathbf{R}^{k}} d y\left|\varphi\left(z_{w, x, y}\right)\right|^{r}\right)^{1 / r}
\end{aligned}
$$

for all $\varphi \in L_{r}\left(\mathbf{R}^{k}\right) \cap L_{2}\left(\mathbf{R}^{k}\right)$ and $\psi \in \mathscr{L}_{q} \cap L_{1}$ whenever $1 / q+1 / r=1$. Then by a change of variables one obtains the bounds

$$
\left\|\psi *_{U} \varphi\right\|_{\infty} \leqq\|\varphi\|_{r}\|\psi \psi\|_{q} .
$$

Therefore if $U_{\psi}$ is defined as an operator from $L_{r}\left(\mathbf{R}^{k}\right) \cap L_{2}\left(\mathbf{R}^{k}\right)$ to $L_{\infty}\left(\mathbf{R}^{k}\right)$ by

$$
U_{\psi} \varphi=\psi *_{U} \varphi
$$

for $\psi \in \mathscr{L}_{q}$ then (10), together with (11), gives bounds

$$
\left\|U_{\psi} \varphi\right\|_{q} \leqq\|\| \psi\|\|_{q}\|\varphi\|_{1}, \quad\left\|U_{\psi} \varphi\right\|_{\infty} \leqq\|\| \psi\|\|_{q}\|\varphi\|_{r} .
$$


Hence $U_{\psi}$ extends to a bounded linear operator from $L_{p_{i}}\left(\mathbf{R}^{k}\right)$ to $L_{q_{i}}\left(\mathbf{R}^{k}\right)$ where $p_{\gamma}^{-1}=\gamma r^{-1}+(1-\gamma), q_{\gamma}^{-1}=(1-\gamma) q^{-1}$ and $\gamma \in[0,1]$. Moreover,

$$
\left\|\psi *_{U} \varphi\right\|_{q_{\gamma}}=\left\|U_{\psi} \varphi\right\|_{q_{\gamma}} \leqq\|\| \psi\|\|_{q}\|\varphi\|_{p_{\gamma}}
$$

by the usual Riesz-Thorin theorem. But $1+q_{\gamma}^{-1}=q^{-1}+p_{\gamma}^{-1}$ and hence one obtains the desired result.

The version of Young's inequalities given in Proposition 3.1 can be used to derive Nash inequalities by the arguments of [Rob], Chapter III, Sect. 3.

Let $b_{1}, \ldots, b_{d^{\prime}}$ be an algebraic basis of $\mathfrak{g}, B_{l}=d U\left(b_{i}\right)$ the representatives on $L_{2}\left(\mathbf{R}^{k}\right)$ and $L_{2 ; n}^{\prime}\left(\mathbf{R}^{k}\right)$ the corresponding $C^{n}$-subspaces (see [Rob], Sect. IV.4). So

$$
L_{2 ; n}^{\prime}\left(\mathbf{R}^{k}\right)=\bigcap_{i_{1}, \ldots, i_{n} \in\left\{1, \ldots, d^{\prime}\right\}} D\left(B_{i_{1}} \cdots B_{i_{n}}\right)
$$

Next let $\rho$ denote the subelliptic distance associated with the basis and $|\cdot|^{\prime}$ the corresponding modulus, i.e., $|g|^{\prime}=\rho(g ; e$ ) (see [Rob], Sect. IV.4). If $\alpha:[0,1] \rightarrow G$ is an absolutely continuous path from the identity $e$ to $g$ with tangents in the space spanned by $b_{1}, \ldots, b_{d^{\prime}}$ then there are $\alpha_{l} \in L_{\infty}([0,1])$ such that

$$
\frac{d \psi(\alpha(t))}{d t}=\sum_{i=1}^{d^{\prime}} \alpha_{i}(t)\left(\tilde{B}_{l} \psi\right)(\alpha(t))
$$

for all $\psi \in C^{\infty}(G)$, where $\tilde{B}_{i}$ is the left invariant vector field on $G$ corresponding to the direction $b_{i}$. We define

$$
|g|^{\prime}=\inf _{\alpha} \int_{0}^{1} d t\left(\sum_{l=1}^{d^{\prime}} \alpha_{t}(t)^{2}\right)^{1 / 2}
$$

where the infimum is over all possible paths. Therefore

$$
\left((I-U(g) \varphi)(x)=\int_{0}^{1} d t \sum_{i=1}^{d^{\prime}} \alpha_{i}(t)\left(U(\alpha(t)) B_{i} \varphi\right)(x)\right.
$$

for all $\varphi \in C_{c}^{\infty}\left(\mathbf{R}^{k}\right)$ and consequently

$$
\|(I-U(g)) \varphi\|_{2} \leqq \int_{0}^{1} d t\left(\sum_{i=1}^{d^{\prime}} \alpha_{i}(t)^{2}\right)^{1 / 2}\left(\sum_{i=1}^{d^{\prime}}\left\|B_{i} \varphi\right\|_{2}^{2}\right)^{1 / 2}
$$

Optimizing this last estimate over the possible paths $\alpha$ one deduces that

$$
\|(I-U(g)) \varphi\|_{2} \leqq|g|^{\prime}\left(\sum_{i=1}^{d^{\prime}}\left\|B_{i} \varphi\right\|_{2}^{2}\right)^{1 / 2}
$$


Therefore if $\psi \in L_{1}(G)$ is a positive function with $\|\psi\|_{1}=1$ one has

$$
\|(I-U(\psi)) \varphi\|_{2} \leqq \int_{G} d g \psi(g)|g|^{\prime}\left(\sum_{l=1}^{d^{\prime}}\left\|B_{l} \varphi\right\|_{2}^{2}\right)^{1 / 2} .
$$

This bound together with Young's inequality now gives the Nash inequality.

Proposition 3.6. Let $a_{1}, \ldots, a_{d_{m}}, \ldots, a_{d_{m}+k}$ be a weak Malcev basis passing through $m$ which has the ideal property (3). For each positive $\psi \in L_{1}(G)$ with $\|\psi\|_{1}=1$ and each algebraic basis $b_{1}, \ldots, b_{d^{\prime}}$ of $\mathfrak{g}$,

$$
\|\varphi\|_{2} \leqq \int_{G} d g \psi(g)|g|^{\prime}\left(\sum_{i=1}^{d^{\prime}}\left\|B_{i} \varphi\right\|_{2}^{2}\right)^{1 / 2}+\|\| \psi\left\|_{2}\right\| \varphi \|_{1}
$$

for all $\varphi \in L_{2 ; 1}^{\prime}\left(\mathbf{R}^{k}\right) \cap L_{1}\left(\mathbf{R}^{k}\right)$. In particular

$$
\|\varphi\|_{2} \leqq \varepsilon\left(\sum_{l=1}^{d^{\prime}}\left\|B_{l} \varphi\right\|_{2}^{2}\right)^{1 / 2}+\left(\|\| \psi_{\varepsilon}\|\|_{2} /\|\| \psi_{\varepsilon} \|_{1}\right)\|\varphi\|_{1},
$$

for each $\varepsilon>0$ where $\psi_{\varepsilon}$ denotes a non-zero, positive, integrable, function with support in the ball $B_{\varepsilon}^{\prime}=\left\{g \in G:|g|^{\prime}<\varepsilon\right\}$.

Proof. First, one has the obvious identity

$$
\|\varphi\|_{2} \leqq\|(I-U(\psi)) \varphi\|_{2}+\|U(\psi) \varphi\|_{2},
$$

and since $U(\psi) \varphi=\psi *_{U} \varphi$ the initial statement of the proposition follows from Proposition 3.1 and (12). The second statement is an immediate consequence of choosing $\psi=\psi_{\varepsilon} /\left\|\psi_{\varepsilon} \mid\right\|_{1}$.

The Nash inequalities (13) can in principle be optimized by minimizing the right-hand side with respect to the choice of $\psi$. The most practical way of tackling this problem appears to be through optimization of (14) with respect to $\varepsilon$ and with $\psi_{\varepsilon}$ a characteristic function. But this requires an efficient bound on $\varepsilon \mapsto\left\||| \chi_{\varepsilon}\right\|_{2} /\left\||| \chi_{\varepsilon}\right\|_{1}$, where $\chi_{\varepsilon}$ is the characteristic function of the ball $B_{\varepsilon}^{\prime}$. The $L_{1}$ norm $\left\|\mid \chi_{\varepsilon}\right\|_{1}$ can be easily estimated because

$$
\left\|\chi_{\varepsilon}\right\|_{1}=\left\|\chi_{\varepsilon}\right\|_{1}=\left|B_{\varepsilon}^{\prime}\right| .
$$

The main problem is to estimate $\left\|\chi_{\varepsilon}\right\|_{2}$. This is straightforward if $b_{1}, \ldots, b_{d^{\prime}}$ is a vector space basis of $g$. Then the corresponding modulus $|g|^{\prime}$ equals the full modulus $|g|$ and the image of $g \mapsto|g|$ under the exponential map is locally equivalent to the Euclidean norm on $\mathbf{R}^{d}$. Hence one has bounds

$$
\alpha^{-1} \varepsilon^{d_{m}+k / 2} \leqq\left\|\chi_{\varepsilon}\right\|_{2} \leqq \alpha \varepsilon^{d_{m}+k / 2}
$$

for some $\alpha>0$ and all $\varepsilon \in\langle 0,1]$. Since one also has estimates $\left\|\left|\chi_{\varepsilon}\right|\right\|_{1} \geqq \alpha^{\prime} \varepsilon^{d}$ for small $\varepsilon$, with $d=d_{\mathrm{m}}+k$ the dimension of the group, this gives bounds

$$
\left\|\chi_{\varepsilon}\right\|_{2} /\left\|\chi_{\varepsilon}\right\|_{1} \leqq \alpha \varepsilon^{-k / 2}
$$

on the ratio which are valid for all $\varepsilon \in\langle 0,1]$. 
For an algebraic basis $b_{1}, \ldots, b_{d^{\prime}}$ the \|\|$\chi_{\varepsilon} \|_{1}$ estimates are clear since one has estimates

$$
\alpha^{-1} \varepsilon^{D^{\prime}} \leqq\left|B_{\varepsilon}^{\prime}\right| \leqq \alpha \varepsilon^{D^{\prime}}
$$

for $\varepsilon \in\langle 0,1]$ and

$$
\alpha_{1}^{-1} \varepsilon^{D} \leqq\left|B_{\varepsilon}^{\prime}\right| \leqq \alpha_{1} \varepsilon^{D}
$$

for $\varepsilon \geqq 1$, for appropriate $\alpha, \alpha_{1}>0$. The two dimensions $D^{\prime}$ and $D$ are usually distinct and $D^{\prime}=D$ if and only if $G$ is stratified and $b_{1}, \ldots, b_{d^{\prime}}$ spans the first subspace in its grading (see [VSC], Remark IV.5.9). The estimation of the $L_{2}$-norm is more difficult.

It is possible to make a crude estimate of $\left\|\chi_{\varepsilon}\right\|_{2}$ for small $\varepsilon$ by remarking that there is a compact subset of $\mathbf{R}^{d}$ which contains the support of the images of $\chi_{\varepsilon}, \varepsilon \in\langle 0,1]$ under the exponential map. Therefore

$$
\alpha_{2}^{-1} \varepsilon^{D^{\prime}} \leqq\left\|\chi_{\varepsilon}\right\|_{2} \leqq \alpha_{2} \varepsilon^{D^{\prime} / 2}
$$

for all $\varepsilon \in\langle 0,1]$ and a suitable $\alpha_{2} \geqq 0$. But a more precise estimate requires more detailed information on the relationship between the Malcev basis $a_{1}, \ldots, a_{d}$ and the algebraic basis $b_{1}, \ldots, b_{d^{\prime}}$. For example, if $G$ is stratified, $b_{1}, \ldots, b_{d^{\prime}}$ is a basis for the first subspace of its grading and each $a_{i}$ is a commutator in the $b_{j}$, then one can find good bounds on $\left\|\chi_{\varepsilon}\right\|_{2}$.

Our inability to establish good estimates on \|\|$\chi_{\varepsilon} \|_{2}$ limits the usefulness of the Nash inequalities for subelliptic operators. Nevertheless, the small $\varepsilon$ estimates (15) yield inequalities which can be usefully applied to the analysis of strongly elliptic operators.

Let $b_{1}, \ldots, b_{d}$ be a vector space basis of $\mathrm{g}$. Then combination of (14) and (15) gives bounds

$$
\|\varphi\|_{2} \leqq \varepsilon\left(\sum_{l=1}^{d}\left\|B_{l} \varphi\right\|_{2}^{2}\right)^{1 / 2}+\alpha \varepsilon^{-k / 2}\|\varphi\|_{1}
$$

for all $\varphi \in L_{2 ; 1}\left(\mathbf{R}^{k}\right) \cap L_{1}\left(\mathbf{R}^{k}\right)$ and all $\varepsilon \in\langle 0,1]$. But if one introduces the norms

$$
\Gamma_{2,1}(\varphi)=\left(\sum_{l=1}^{d}\left\|B_{i} \varphi\right\|_{2}^{2}+\gamma^{2}\|\varphi\|_{2}^{2}\right)^{1 / 2}
$$

on $L_{2 ; 1}\left(\mathbf{R}^{k}\right)$ with $\gamma \in\langle 0,1]$ one then has bounds

$$
\|\varphi\|_{2} \leqq \varepsilon \Gamma_{2 ; 1}(\varphi)+\alpha \varepsilon^{-k / 2}\|\varphi\|_{1}
$$

valid for all $\varepsilon \in\langle 0,1]$ and for $\varepsilon \geqq 1 / \gamma$. But these bounds can be simply modified to hold for all $\varepsilon>0$ and then optimized over $\varepsilon$.

Corollary 3.7. Let $a_{1}, \ldots, a_{d_{m}}, \ldots, a_{d_{m}+k}$ be a weak Malcev basis passing through $\mathrm{m}$ with the ideal property (3) and let $b_{1}, \ldots, b_{d}$ a vector space basis for $\mathrm{g}$. Then there is an $\alpha>0$ such that

$$
\|\varphi\|_{2} \leqq \varepsilon \Gamma_{2 ; 1}(\varphi)+\alpha(\gamma \varepsilon)^{-k / 2}\|\varphi\|_{1}
$$


for all $\varphi \in L_{2 ; 1} \cap L_{1}$, all $\varepsilon>0$ and all $\gamma \in\langle 0,1]$. Consequently, there is an $\alpha_{1}>0$ such that

$$
\|\varphi\|_{2} \leqq \alpha_{1}\left(\Gamma_{2 ; 1}(\varphi) /\left(\gamma\|\varphi\|_{1}\right)\right)^{k /(k+2)}\|\varphi\|_{1}
$$

for all $\varphi \in L_{2,1} \cap L_{1}$ and all $\gamma \in\langle 0,1]$.

Remark 3.8. The above Nash inequalities are expressed, or are expressable, in terms of the $C^{1}$-seminorms $N_{2,1}$, or the $C^{1}$-norms $\|\cdot\|_{2,1}$ used in [Rob]. Similar results can, however, be formulated with the $C^{n}$-seminorms and $C^{n}$-norms by the use of embedding properties. In particular for each $n \in\{2,3, \ldots\}$ there is an $\alpha_{n}>0$ such that

$$
N_{2 ; 1}(\varphi) \leqq \varepsilon^{n-1} N_{2 ; n}(\varphi)+\alpha_{n} \varepsilon^{-1}\|\varphi\|_{2}
$$

for all $\varphi \in L_{2 ; n}$ and all $\varepsilon \in\langle 0,1]$ (see [Rob], Lemma III.3.3). Similarly,

$$
\|\varphi\|_{2,1} \leqq \varepsilon^{n-1}\|\varphi\|_{2 ; n}+\alpha_{n}^{\prime} \varepsilon^{-1}\|\varphi\|_{2}
$$

for all $\varphi \in L_{2, n}$ and all $\varepsilon>0$.

In the sequel we need a variation of the above results which is formulated in terms of a second representation $U^{\circ}$ of $G$ associated with $U$. The action of $U$ is given by (1) which can be reformulated with the notation of (2) as

$$
(U(g) \varphi)(x)=e^{I \sigma_{g}(x)} \varphi\left(\theta_{g}(x)\right),
$$

and then the action of $U^{\circ}$ is defined by

$$
\left(U^{\circ}(g) \varphi\right)(x)=\varphi\left(\theta_{g}(x)\right) .
$$

It then follows as for $U$ that $U^{\circ}$ is an isometric continuous representation on $L_{p}\left(\mathbf{R}^{k}\right)$ for $p \in[1, \infty]$. Note that if $b \in \mathfrak{g}$ and $B=d U(b)$ then

$$
(B \varphi)(x)=\sum_{n=1}^{k} X_{n}(x) \frac{\partial \varphi}{\partial x_{n}}(x)+i Y(x) \varphi(x)
$$

with $X_{n}$ and $Y$ real polynomials. Hence if $B^{\circ}=d U^{\circ}(b)$ one has

$$
\left(B^{\circ} \varphi\right)(x)=\sum_{n=1}^{k} X_{n}(x) \frac{\partial \varphi}{\partial x_{n}}(x),
$$

i.e., $B^{\circ}$ is the principal part of the first-order partial differential operator $B$.

Now if one defines a convolution product $\psi *_{U} \circ \varphi$ by setting

$$
\psi *_{U} \circ \varphi=U^{\circ}(\psi) \varphi
$$

then the generalized Young inequality is again valid.

Proposition 3.9. Let $a_{1}, \ldots, a_{d_{m}}, \ldots, a_{d_{m}+k}$ be a weak Malcev basis passing through $m$ which has the ideal property (3). If $p, q, r \in[1, \infty]$ and $1+1 / r=1 / p+1 / q$ then $(\psi, \varphi) \mapsto \psi *_{U} \circ \varphi$ from $\left(\mathscr{L}_{q} \cap \mathscr{L}_{1}\right) \times\left(L_{p}\left(\mathbf{R}^{k}\right) \cap L_{2}\left(\mathbf{R}^{k}\right)\right)$ into $L_{r}\left(\mathbf{R}^{k}\right)$ extends to a map from $\mathscr{L}_{q} \times L_{p}\left(\mathbf{R}^{k}\right)$ into $L_{r}\left(\mathbf{R}^{k}\right)$ which satisfies

$$
\left\|\psi *_{U} \circ \varphi\right\|_{r} \leqq\|\varphi\|_{p}\|\| \psi \|_{q}
$$

for all $\varphi \in L_{p}\left(\mathbf{R}^{k}\right)$ and $\psi \in \mathscr{L}_{q}$. 
Proof. The proof is very similar to that for the representation $U$ but the starting point is now the identity

$$
\left(U^{\circ}(\psi) \varphi\right)(x)=\int_{\mathbf{R}^{d_{m}}} d w \int_{\mathbf{R}^{k}} d y \psi(\beta(w) \gamma(y)) \varphi\left(z_{w, x, y}\right),
$$

which then gives

$$
\left|\left(U^{\circ}(\psi) \varphi\right)(x)\right| \leqq \int_{\mathbf{R}^{d_{m}}} d w \int_{\mathbf{R}^{k}} d y|\psi(\beta(w) \gamma(y))| \cdot\left|\varphi\left(z_{w, x, y}\right)\right|
$$

in direct analogy with (8). The point is that the phase which distinguishes between the action of $U$ and $U^{\circ}$ plays no role in this estimate or in the subsequent estimates that are essential in the proof.

One can now derive a version of the Nash inequalities suited to the seminorms associated with the operators $B_{l}^{\circ}=d U^{\circ}\left(b_{i}\right)$. One has

$$
\left\|\left(I-U^{\circ}(\psi)\right) \varphi\right\|_{2} \leqq \int_{G} d g \psi(g)|g|\left(\sum_{i=1}^{d}\left\|B_{l}^{\circ} \varphi\right\|_{2}^{2}\right)^{1 / 2}
$$

in direct analogy with (12). Therefore if

$$
\Gamma_{2 ; 1}^{\circ}(\varphi)=\left(\sum_{i=1}^{d}\left\|B_{l}^{\circ} \varphi\right\|_{2}^{2}+\gamma^{2}\|\varphi\|_{2}^{2}\right)^{1 / 2}
$$

with $\gamma \in\langle 0,1]$ one obtains the following version of Corollary 3.7.

Corollary 3.10. Let $a_{1}, \ldots, a_{d_{m}}, \ldots, a_{d_{m}+k}$ be a weak Malcev basis passing through $m$ with the ideal property (3) and let $b_{1}, \ldots, b_{d}$ a vector space basis for $\mathrm{g}$. Then there is an $\alpha>0$ such that

$$
\|\varphi\|_{2} \leqq \varepsilon \Gamma_{2 ; 1}^{\circ}(\varphi)+\alpha(\gamma \varepsilon)^{-k / 2}\|\varphi\|_{1}
$$

for all $\varphi \in L_{2 ; 1} \cap L_{1}$, all $\varepsilon>0$ and all $\gamma \in\langle 0,1]$. Consequently, there is an $\alpha_{1}>0$ such that

$$
\|\varphi\|_{2} \leqq \alpha_{1}\left(\Gamma_{2 ; 1}^{\circ}(\varphi) /\left(\gamma\|\varphi\|_{1}\right)\right)^{k /(k+2)}\|\varphi\|_{1}
$$

for all $\varphi \in L_{2 ; 1} \cap L_{1}$ and all $\gamma \in\langle 0,1]$.

The proof is a repetition of the previous arguments but with Proposition 3.1 replaced by Proposition 3.9.

\section{Kernel Bounds: Small $t$}

In this section we use the Nash inequalities to obtain bounds on the reduced kernel $\kappa_{t}$ associated with the strongly elliptic semigroup $S_{t}$. Since the Nash inequalities are established for weak Malcev bases with the ideal property (3) we first derive kernel bounds in a representation realized with respect to such a basis. Subsequently we remove the ideal property by making a unitary transformation.

Our arguments are based on the Davies perturbation method as described in [Rob] Sect. IV.2. A complication occurs, however, since the present operators are 
in general not real. Therefore we have to work on the complex $L_{p}\left(\mathbf{R}^{k}\right)$ spaces. One cannot restrict attention to the subspaces spanned by the real-valued functions as in [Rob].

Let $U$ be a basis realization on $L_{2}\left(\mathbf{R}^{k}\right)$ of the induced representation $\pi$ and $b_{1}, \ldots, b_{d}$ a (vector space) basis of the Lie algebra g. If $B_{J}=d U\left(b_{J}\right)$ for $j \in$ $\{1, \ldots, d\}$, then

$$
\left(B_{j} \varphi\right)(x)=\sum_{n=1}^{k} X_{j n}(x) \frac{\partial \varphi}{\partial x_{n}}(x)+i Y_{j}(x) \varphi(x)
$$

with $X_{j n}$ and $Y_{j}$ real polynomials. Moreover, if $U^{\circ}$ is the representation of $G$ defined at the end of Sect. 3 , then $B_{j}^{\circ}=d U^{\circ}\left(b_{j}\right)$ is the principal part of $B_{j}$, i.e.,

$$
\left(B_{,}^{\circ} \varphi\right)(x)=\sum_{n=1}^{k} X_{j n}(x) \frac{\partial \varphi}{\partial x_{n}}(x) .
$$

Next, if $C=\left(c_{l j}\right)$ is a real, symmetric, strictly positive-definite matrix we define

$$
D_{C}=\left\{\psi \in C_{c}^{\infty}\left(\mathbf{R}^{k}\right): \psi \text { real valued and } \sum_{i, j=1}^{d} c_{l j}\left(B_{l}^{\circ} \psi\right)(x)\left(B_{J}^{\circ} \psi\right)(x) \leqq 1\right.
$$

$$
\text { for all } \left.x \in \mathbf{R}^{k}\right\} \text {, }
$$

and then the distance $d_{U, C}: \mathbf{R}^{k} \times \mathbf{R}^{k} \rightarrow[0, \infty\rangle$ is introduced by

$$
d_{U, C}(x ; y)=\sup _{\psi \in D_{C}}|\psi(x)-\psi(y)| .
$$

The first theorem of this section gives kernel bounds for second-order operators

$$
H=-\sum_{l, j=1}^{d} c_{i j} B_{i} B_{j}+\sum_{i=1}^{d} c_{i} B_{i}
$$

with the matrix $C$ as principal coefficients and with real first-order coefficients $c_{i}$. The large time behaviour of the bounds is governed by the smallest eigenvalue $\lambda_{1}$ of the self-adjoint principal part

$$
H_{0}=-\sum_{i, j=1}^{d} c_{l j} B_{i} B_{J}
$$

of $H$ acting on $L_{2}\left(\mathbf{R}^{k}\right)$, i.e.,

$$
\begin{aligned}
\lambda_{1} & =\min \left\{\left(\varphi, H_{0} \varphi\right): \varphi \in \mathscr{S}\left(\mathbf{R}^{k}\right) \text { and }\|\varphi\|_{2}=1\right\} \\
& =\min \left\{(\varphi, H \varphi): \varphi \in \mathscr{S}\left(\mathbf{R}^{k}\right) \text { and }\|\varphi\|_{2}=1\right\} .
\end{aligned}
$$

Note that $\lambda_{1}>0$ since $k \geqq 1$. Indeed if $\lambda_{1}=0$ then the corresponding normalized eigenfunction $\varphi_{1}$ would satisfy

$$
\sum_{i, j=1}^{d} c_{i j}\left(B_{l} \varphi_{1}, B_{J} \varphi_{1}\right)=0
$$


and, since $C$ is strictly positive, $B_{i} \varphi_{1}=0$ for all $i \in\{1, \ldots, d\}$. But this implies that $U(g) \varphi_{1}=\varphi_{1}$ for all $g \in G$ which is impossible since $U$ is irreducible and non-trivial. Further note that $\lambda_{1}$ is a unitary invariant, i.e., if $U$ and $\widetilde{U}$ are unitarily equivalent representations, with $H, \widetilde{H}$ the corresponding strongly elliptic operators and $\lambda_{1}, \tilde{\lambda}_{1}$ the lowest eigenvalues then $\lambda_{1}=\tilde{\lambda}_{1}$. This invariance will play a minor role in the following proof.

Theorem 4.1. Let $l \in \mathrm{g}^{*}, a_{1}, \ldots, a_{d_{\mathfrak{m}}}, \ldots, a_{d_{\mathrm{m}}+k}$ be a weak Malcev basis passing through a polarizing subalgebra $\mathrm{m}$ of $l$ and $U$ the corresponding basis realization in $L_{2}\left(\mathbf{R}^{k}\right)$. Let $H$ be a second-order operator associated with the real, symmetric, strictly positive-definite matrix $C=\left(c_{i j}\right)$, the first-order coefficients $c_{l} \in \mathbf{R}$ and the basis $b_{1}, \ldots, b_{d}$ of $\mathfrak{g}$. Further let $\kappa_{t}$ denote the corresponding reduced kernel. Then there exists an $a>0$, independent of the coefficients $(C, c)$, such that

$$
\left|\kappa_{t}(x ; y)\right| \leqq a(1 \wedge \varepsilon \mu t)^{-k / 2} e^{-\lambda_{1} t} \inf _{\rho \geqq 0} \exp \left(\rho^{2}(1+\varepsilon) t-\rho\left(d_{U, C}(x ; y)-v t\right)\right)
$$

uniformly for all $t>0, x, y \in \mathbf{R}^{k}$ and $\varepsilon \in\langle 0,1]$, where $\mu$ is the lowest eigenvalue of $C$,

$$
\lambda_{1}=\min \left\{(\varphi, H \varphi): \varphi \in \mathscr{S}\left(\mathbf{R}^{k}\right) \text { and }\|\varphi\|_{2}=1\right\}
$$

and $v=|c| \mu^{-1 / 2}$ with $|c|$ the $l_{2}$-norm of the first-order coefficients.

Therefore if $d_{U, C}(x ; y) \leqq v t$ then

$$
\left|\kappa_{t}(x ; y)\right| \leqq a(1 \wedge \varepsilon \mu t)^{-k / 2} e^{-\lambda_{1} t}
$$

and if $d_{U, C}(x ; y) \geqq v t$ then

$$
\left|\kappa_{t}(x ; y)\right| \leqq a(1 \wedge \varepsilon \mu t)^{-k / 2} e^{-\lambda_{1} t} \exp \left(-\left(d_{U, C}(x ; y)-v t\right)^{2}(4(1+\varepsilon) t)^{-1}\right)
$$

for all $\varepsilon \in\langle 0,1]$.

This result is the direct analogue of Theorem IV.2.2 for the universal kernel given in [Rob]. The proof is very similar although the complex structure introduces added complications.

These bounds on the reduced kernel give the optimal $t$-singularity for small $t$ and the correct asymptotic behaviour for large $t$. In particular

$$
\lim _{t \rightarrow \infty}-t^{-1} \log \left|\kappa_{t}(x ; y)\right| \geqq \lambda_{1} .
$$

In addition the bounds give

$$
\lim _{t \rightarrow 0}-t \log \left|\kappa_{t}(x ; y)\right| \geqq d_{U, C}(x ; y)^{2} / 4,
$$

which is the optimal bound in the relative variable. (It is likely that both these bounds are identities.)

The principal weakness of the kernel bounds is that they fail to reflect the expected exponential decrease of the kernel on the diagonal. This will be established in the next section by an alternative set of bounds.

Proof. We begin by assuming that the weak Malcev basis has the ideal property (3). 
Let $\psi \in D_{C}$. For $\rho \in \mathbf{R}$ define the operator $U_{\rho}$ on $L_{2}\left(\mathbf{R}^{k}\right)$ by $\left(U_{\rho} \varphi\right)(x)=$ $e^{-\rho \psi(x)} \varphi(x)$ and the semigroup $S^{\rho}$ by $S_{t}^{\rho}=U_{\rho} S_{t} U_{\rho}^{-1}$. Then the infinitesimal generator of $S^{\rho}$ is the operator $H^{\rho}=U_{\rho} H U_{\rho}^{-1}$. Note that $U_{\rho} B_{i} U_{\rho}^{-1} \varphi=B_{i} \varphi+\psi_{i} \varphi$ for all $\varphi \in L_{2}\left(\mathbf{R}^{k}\right)$, where $\psi_{i}=B_{l}^{\circ} \psi$.

Let $\rho \in \mathbf{R}, \varphi \in L_{2}\left(\mathbf{R}^{k}\right)$ and set $\varphi_{t}=S_{t}^{\rho} \varphi$ for all $t>0$. Then for all $t>0$ one has

$$
\begin{aligned}
\frac{d}{d t}\left\|\varphi_{t}\right\|_{2}^{2} & =-2 \operatorname{Re}\left(\varphi_{t}, H^{\rho} \varphi_{t}\right) \\
& =-2 \operatorname{Re} \sum_{i, j=1}^{d} c_{i j}\left(\left(B_{i}-\rho \psi_{l}\right) \varphi_{t},\left(B_{j}+\rho \psi_{J}\right) \varphi_{t}\right)-2 \operatorname{Re} \sum_{l=1}^{d} c_{l}\left(\varphi_{t},\left(B_{l}+\rho \psi_{i}\right) \varphi_{t}\right) \\
& =-2\left(\varphi_{t}, H_{0} \varphi_{t}\right)+2 \rho^{2} \sum_{i, j=1}^{d} c_{i j}\left(\psi_{i} \varphi_{t}, \psi_{j} \varphi_{t}\right)-2 \rho \sum_{t=1}^{d} c_{l}\left(\varphi_{t}, \psi_{l} \varphi_{t}\right) \\
& \leqq 2\left(\rho^{2}-\lambda_{1}+|\rho| v\right)\left\|\varphi_{t}\right\|_{2}^{2} .
\end{aligned}
$$

(Here we have used the estimate

$$
\sum_{i=1}^{d}\left\|\psi_{l} \varphi\right\|_{2}^{2} \leqq \mu^{-1} \sum_{l, j=1}^{d} c_{l j}\left(\psi_{l} \varphi, \psi_{J} \varphi\right) \leqq \mu^{-1}\|\varphi\|_{2}^{2}
$$

which is valid for all $\varphi \in L_{2}\left(\mathbf{R}^{k}\right)$.) Hence by integration one finds

$$
\left\|S_{t}^{\rho}\right\|_{2 \rightarrow 2} \leqq e^{\left(\rho^{2}-\lambda_{1}+|\rho| v\right) t}
$$

for all $t>0$.

Next we estimate $\left\|S_{t}^{\rho}\right\|_{2 \rightarrow \infty}$. Let $\rho \in \mathbf{R}$ and $\varphi \in \bigcap_{p=1}^{\infty} L_{p}$. Then $\varphi_{t}=S_{t}^{\rho} \varphi \in$ $\mathscr{S}\left(\mathbf{R}^{k}\right) \subset \bigcap_{p=1}^{\infty} L_{p}$. Thus if $p \geqq 2$ is an even integer,

$\frac{d}{d t}\left\|\varphi_{t}\right\|_{2 p}^{2 p}=-2 p \operatorname{Re}\left(\varphi_{t}^{p}, \varphi_{t}^{p-1} H^{\rho} \varphi_{t}\right)$

$$
\begin{aligned}
= & -2 p \operatorname{Re} \sum_{l, j=1}^{d} c_{l j}\left(B_{i}\left(\varphi_{t}^{p}{\overline{\varphi_{t}}}^{p-1}\right), B_{j} \varphi_{t}\right)+2 p \rho \operatorname{Re} \sum_{l, j=1}^{d} c_{i j}\left(\psi_{i} \varphi_{t}^{p}{\overline{\varphi_{t}}}^{p-1}, B_{j} \varphi_{t}\right) \\
& -2 p \rho \operatorname{Re} \sum_{i, j=1}^{d} c_{l j}\left(B_{l}\left(\varphi_{t}^{p}{\overline{\varphi_{t}}}^{p-1}\right), \psi_{j} \varphi_{t}\right)+2 p \rho^{2} \operatorname{Re} \sum_{l, j=1}^{d} c_{l j}\left(\psi_{i} \varphi_{t}^{p}{\overline{\varphi_{t}}}^{p-1}, \psi_{j} \varphi_{t}\right) \\
& -2 p \operatorname{Re} \sum_{i=1}^{d} c_{l}\left(\varphi_{t}^{p}{\overline{\varphi_{t}}}^{p-1}, B_{l} \varphi_{t}\right)-2 p \rho \operatorname{Re} \sum_{t=1}^{d} c_{i}\left(\varphi_{t}^{p}{\overline{\varphi_{t}}}^{p-1}, \psi_{i} \varphi_{t}\right) .
\end{aligned}
$$

We estimate the six terms separately. Using the identity $B_{i}(\varphi \psi)=\psi B_{1}^{\circ} \varphi+$ $\varphi B_{l} \psi$, together with the fact that $B_{i}^{\circ}$ is a derivation, one obtains for the first term

$$
\begin{aligned}
\left(B_{l}\left(\varphi_{t}^{p}{\overline{\varphi_{t}}}^{p-1}\right), B_{J} \varphi_{t}\right)=\left(B_{l}\left(\left(\left|\varphi_{t}\right|^{2}\right)^{p-1} \varphi_{t}\right), B_{j} \varphi_{t}\right) \\
=(p-1)\left(\left|\varphi_{t}\right|^{2 p-4} \varphi_{t} B_{l}^{\circ}\left|\varphi_{t}\right|^{2}, B_{j} \varphi_{t}\right)+\left(\left|\varphi_{t}\right|^{2 p-2} B_{l} \varphi_{t}, B_{j} \varphi_{t}\right) \\
=(p-1)\left(\left|\varphi_{t}\right|^{2 p-4} B_{i}^{\circ}\left|\varphi_{t}\right|^{2}, \chi_{j}\right) \\
\quad+i(p-1)\left(\left|\varphi_{t}\right|^{2 p-4} B_{i}^{\circ}\left|\varphi_{t}\right|^{2}, Y_{J}\left|\varphi_{t}\right|^{2}\right)+\left(\left|\varphi_{t}\right|^{p-1} B_{i} \varphi_{t},\left|\varphi_{t}\right|^{p-1} B_{j} \varphi_{t}\right),
\end{aligned}
$$


where $\chi=\left(\overline{\varphi_{t}} B_{1}^{\circ} \varphi_{t}, \ldots, \overline{\varphi_{t}} B_{d}^{\circ} \varphi_{t}\right)$. The key point is that the second term is purely imaginary since $B_{i}^{\circ}$ is a real differential operator. Moreover, $\left|\varphi_{t}\right|^{2 p-4} B_{i}^{\circ}\left|\varphi_{t}\right|^{2}$ is real. Hence using the identity $\chi_{j}+\overline{\chi_{j}}=B_{j}^{\circ}\left|\varphi_{t}\right|^{2}$ one deduces that

$$
\begin{aligned}
- & 2 p \operatorname{Re} \sum_{i, j=1}^{d} c_{l j}\left(B_{l}\left(\varphi_{t}^{p} \overline{\varphi_{t}}{ }^{p-1}\right), B_{J} \varphi_{t}\right) \\
= & -p(p-1) \sum_{l, j=1}^{d} c_{l j}\left(\left|\varphi_{t}\right|^{2 p-4} B_{i}^{\circ}\left|\varphi_{t}\right|^{2}, \chi_{j}+\overline{\chi_{j}}\right) \\
& -2 p \sum_{l, j=1}^{d} c_{i j}\left(\left|\varphi_{t}\right|^{p-1} B_{i} \varphi_{t},\left|\varphi_{t}\right|^{p-1} B_{j} \varphi_{t}\right) \\
= & -p(p-1) \sum_{i, j=1}^{d} c_{i j}\left(\left|\varphi_{t}\right|^{2 p-4} B_{i}^{\circ}\left|\varphi_{t}\right|^{2}, B_{j}^{\circ}\left|\varphi_{t}\right|^{2}\right) \\
& -2 p \sum_{i, j=1}^{d} c_{l j}\left(\left|\varphi_{t}\right|^{p-1} B_{l} \varphi_{t},\left|\varphi_{t}\right|^{p-1} B_{j} \varphi_{t}\right) \\
= & -4 p^{-1}(p-1) \sum_{i, j=1}^{d} c_{i j}\left(B_{l}^{\circ}\left|\varphi_{t}\right|^{p}, B_{j}^{\circ}\left|\varphi_{t}\right|^{p}\right)-2 p \sum_{i, j=1}^{d} c_{i j}\left(\left|\varphi_{t}\right|^{p-1} B_{l} \varphi_{t},\left|\varphi_{t}\right|^{p-1} B_{J} \varphi_{t}\right) \\
\leqq & -2 \sum_{l, j=1}^{d} c_{i j}\left(B_{i}^{\circ}\left|\varphi_{t}\right|^{p}, B_{j}^{\circ}\left|\varphi_{t}\right|^{p}\right)-2 p \sum_{l, j=1}^{d} c_{l j}\left(\left|\varphi_{t}\right|^{p-1} B_{l} \varphi_{t},\left|\varphi_{t}\right|^{p-1} B_{J} \varphi_{t}\right)
\end{aligned}
$$

because $p \geqq 2$.

Next we consider the second order terms on the right-hand side of (17) which are proportional to $\rho$. One has

$$
\begin{aligned}
2 p\left|\rho \operatorname{Re} \sum_{i, J=1}^{d} c_{l j}\left(\psi_{l} \varphi_{t}^{p}{\overline{\varphi_{t}}}^{p-1}, B_{j} \varphi_{t}\right)\right| & =2 p|\rho|\left|\operatorname{Re} \sum_{l, j=1}^{d} c_{l j}\left(\psi_{i}\left|\varphi_{t}\right|^{p-1} \varphi_{t},\left|\varphi_{t}\right|^{p-1} B_{j} \varphi_{t}\right)\right| \\
\leqq & \varepsilon p|\rho| \sum_{i, j=1}^{d} c_{l j}\left(\left|\varphi_{t}\right|^{p-1} B_{l} \varphi_{t},\left|\varphi_{t}\right|^{p-1} B_{j} \varphi_{t}\right) \\
& +\varepsilon^{-1} p|\rho| \sum_{i, j=1}^{d} c_{i j}\left(\psi_{i}\left|\varphi_{t}\right|^{p-1} \varphi_{t}, \psi_{j}\left|\varphi_{t}\right|^{p-1} \varphi_{t}\right) .
\end{aligned}
$$

Therefore choosing $\varepsilon=(2|\rho|)^{-1}$ one finds

$$
\begin{aligned}
2 p\left|\rho \operatorname{Re} \sum_{i, j=1}^{d} c_{i j}\left(\psi_{i} \varphi_{t}^{p} \overline{\bar{\varphi}_{t}}{ }^{p-1}, B_{j} \varphi_{t}\right)\right| \leqq & 2^{-1} p \sum_{i, j=1}^{d} c_{i j}\left(\left|\varphi_{t}\right|^{p-1} B_{i} \varphi_{t},\left|\varphi_{t}\right|^{p-1} B_{j} \varphi_{t}\right) \\
& +2 p \rho^{2}\left\|\varphi_{t}^{p}\right\|_{2}^{2} .
\end{aligned}
$$

Alternatively,

$$
\begin{aligned}
\left(B_{i}\left(\varphi_{t}^{p}{\overline{\varphi_{t}}}^{p-1}\right), \psi_{j} \varphi_{t}\right) & =(p-1)\left(\left|\varphi_{t}\right|^{2 p-4} \varphi_{t} B_{l}^{\circ}\left|\varphi_{t}\right|^{2}, \psi_{j} \varphi_{t}\right)+\left(\left|\varphi_{t}\right|^{2 p-2} B_{i} \varphi_{t}, \psi_{j} \varphi_{t}\right) \\
& =2 p^{-1}(p-1)\left(B_{l}^{\circ}\left|\varphi_{t}\right|^{p}, \psi_{j}\left|\varphi_{t}\right|^{p}\right)+\left(\left|\varphi_{t}\right|^{p-1} B_{i} \varphi_{t},\left|\varphi_{t}\right|^{p-1} \varphi_{t} \psi_{J}\right) .
\end{aligned}
$$


Hence estimating as before

$$
\begin{aligned}
& 2 p\left|\rho \operatorname{Re} \sum_{i, j=1}^{d} c_{l j}\left(B_{i}\left(\varphi_{t}^{p}{\overline{\varphi_{t}}}^{p-1}\right), \psi_{j} \varphi_{t}\right)\right| \leqq 2 \varepsilon(p-1)|\rho| \sum_{i, j=1}^{d} c_{i j}\left(B_{l}^{\circ}\left|\varphi_{t}\right|^{p}, B_{j}^{\circ}\left|\varphi_{t}\right|^{p}\right) \\
& \quad+2 \varepsilon^{-1}(p-1)|\rho| \sum_{i, j=1}^{d} c_{i j}\left(\psi_{i}\left|\varphi_{t}\right|^{p}, \psi_{j}\left|\varphi_{t}\right|^{p}\right) \\
& \quad+\delta p|\rho| \sum_{l, J=1}^{d} c_{i j}\left(\left|\varphi_{t}\right|^{p-1} B_{i} \varphi_{t},\left|\varphi_{t}\right|^{p-1} B_{j} \varphi_{t}\right) \\
& \quad+\delta^{-1} p|\rho| \sum_{i, j=1}^{d} c_{i j}\left(\psi_{i}\left|\varphi_{t}\right|^{p-1} \varphi_{t}, \psi_{j}\left|\varphi_{t}\right|^{p-1} \varphi_{t}\right) .
\end{aligned}
$$

Therefore choosing $\delta=(2|\rho|)^{-1}$ and $\varepsilon=(2(p-1)|\rho|)^{-1}$ one concludes that

$$
\begin{aligned}
& 2 p\left|\rho \operatorname{Re} \sum_{l, j=1}^{d} c_{i j}\left(B_{i}\left(\varphi_{t}^{p} \overline{\bar{\varphi}_{t}}{ }^{p-1}\right), \psi_{J} \varphi_{t}\right)\right| \\
& \leqq \sum_{l, j=1}^{d} c_{l j}\left(B_{l}^{\circ}\left|\varphi_{t}\right|^{p}, B_{J}^{\circ}\left|\varphi_{t}\right|^{p}\right)+4(p-1)^{2} \rho^{2}\left\|\varphi_{t}^{p}\right\|_{2}^{2} \\
& \quad+2^{-1} p \sum_{l, j=1}^{d} c_{l J}\left(\left|\varphi_{t}\right|^{p-1} B_{i} \varphi_{t},\left|\varphi_{t}\right|^{p-1} B_{J} \varphi_{t}\right)+2 p \rho^{2}\left\|\varphi_{t}^{p}\right\|_{2}^{2} .
\end{aligned}
$$

The fourth term on the right-hand side of (17) is straightforwardly estimated,

$$
2 p \rho^{2}\left|\operatorname{Re} \sum_{i, j=1}^{d} c_{i j}\left(\psi_{i} \varphi_{t}^{p}{\overline{\varphi_{t}}}^{p-1}, \psi_{j} \varphi_{t}\right)\right| \leqq 2 p \rho^{2}\left\|\varphi_{t}^{p}\right\|_{2}^{2} .
$$

For the fifth term we use the skew-adjointness of $B_{l}$ and $B_{i}^{\circ}$ to deduce that

$$
\begin{aligned}
& -2 p \operatorname{Re} \sum_{l=1}^{d} c_{l}\left(\varphi_{t}^{p} \overline{\varphi_{t}}{ }^{p-1}, B_{l} \varphi_{t}\right)=-2 p \operatorname{Re} \sum_{i=1}^{d} c_{i}\left(\left|\varphi_{t}\right|^{2 p-2} \varphi_{t}, B_{l} \varphi_{t}\right) \\
& =2 p \operatorname{Re} \sum_{i=1}^{d} c_{i}\left(B_{i}\left(\left|\varphi_{t}\right|^{2 p-2} \varphi_{t}\right), \varphi_{t}\right) \\
& =2 p \operatorname{Re} \sum_{l=1}^{d} c_{l}\left(\left|\varphi_{t}\right|^{2 p-2} B_{i} \varphi_{t}, \varphi_{t}\right)+2(2 p-2) \operatorname{Re} \sum_{i=1}^{d} c_{l}\left(\left|\varphi_{t}\right|^{p-2} \varphi_{t} B_{l}^{\circ}\left|\varphi_{t}\right|^{p}, \varphi_{t}\right) \\
& =2 p \operatorname{Re} \sum_{i=1}^{d} c_{l}\left(\left|\varphi_{t}\right|^{2 p-2} B_{l} \varphi_{t}, \varphi_{t}\right)+2(2 p-2) \operatorname{Re} \sum_{i=1}^{d} c_{l}\left(B_{l}^{\circ}\left|\varphi_{t}\right|^{p},\left|\varphi_{t}\right|^{p}\right) \\
& =2 p \operatorname{Re} \sum_{l=1}^{d} c_{l}\left(\left|\varphi_{t}\right|^{2 p-2} B_{l} \varphi_{t}, \varphi_{t}\right) .
\end{aligned}
$$

Therefore one concludes that

$$
-2 p \operatorname{Re} \sum_{i=1}^{d} c_{i}\left(\varphi_{t}^{p}{\overline{\varphi_{t}}}^{p-1}, B_{l} \varphi_{t}\right)=0 .
$$


Finally,

$$
2 p\left|\rho \operatorname{Re} \sum_{i=1}^{d} c_{l}\left(\varphi_{t}^{p}{\overline{\varphi_{t}}}^{p-1}, \psi_{i} \varphi_{t}\right)\right|=2 p\left|\rho \sum_{l=1}^{d} c_{l}\left(\left|\varphi_{t}\right|^{p}, \psi_{i}\left|\varphi_{t}\right|^{p}\right)\right| \leqq 2 p|\rho| v\left\|\varphi_{t}^{p}\right\|_{2}^{2} .
$$

Adding all these terms one derives the differential inequality

$$
\begin{aligned}
\frac{d}{d t}\left\|\varphi_{t}\right\|_{2 p}^{2 p} \leqq & -\sum_{l, j=1}^{d} c_{l j}\left(B_{i}^{\circ}\left|\varphi_{t}\right|^{p}, B_{j}^{\circ}\left|\varphi_{t}\right|^{p}\right)-p \sum_{l, j=1}^{d} c_{i j}\left(\left|\varphi_{t}\right|^{p-1} B_{l} \varphi_{t},\left|\varphi_{t}\right|^{p-1} B_{j} \varphi_{t}\right) \\
& +\left(4 p^{2} \rho^{2}+2 p|\rho| v\right)\left\|\varphi_{t}^{p}\right\|_{2}^{2} \\
\leqq & -\mu \sum_{i=1}^{d}\left\|B_{i}^{\circ}\left|\varphi_{t}\right|^{p}\right\|_{2}^{2}+\left(4 p^{2} \rho^{2}+2 p|\rho| v\right)\left\|\varphi_{t}^{p}\right\|_{2}^{2} .
\end{aligned}
$$

Now using $\left\|\varphi_{t}^{p}\right\|_{2}^{2}=\left\|\varphi_{t}\right\|_{2 p}^{2 p}$ one obtains

$$
\frac{d}{d t}\left\|\varphi_{t}\right\|_{2 p} \leqq-\mu(2 p)^{-1}\left\|\varphi_{t}\right\|_{2 p}^{1-2 p} \sum_{l=1}^{d}\left\|B_{l}^{\circ}\left|\varphi_{t}\right|^{p}\right\|_{2}^{2}+\left(2 p \rho^{2}+|\rho| v\right)\left\|\varphi_{t}\right\|_{2 p} .
$$

Finally, in terms of the norm $\Gamma_{2 ; 1}^{\circ}$ introduced in Sect. 3,

$$
\frac{d}{d t}\left\|\varphi_{t}\right\|_{2 p} \leqq-\mu(2 p)^{-1}\left\|\varphi_{t}\right\|_{2 p}^{1-2 p} \Gamma_{2,1}^{\circ}\left(\left|\varphi_{t}\right|^{p}\right)^{2}+\left(2 p \rho^{2}+|\rho| v+\gamma^{2} \mu(2 p)^{-1}\right)\left\|\varphi_{t}\right\|_{2 p} .
$$

This differential inequality is the same as inequality (IV.2.12) in [Rob], if one takes $\|C\|=1$ in [Rob]. The important feature of the remaining part of the proof is the use of the Nash inequalities of Corollary 3.10 to estimate the terms in the sum. These estimates are in terms of $L_{1^{-}}$, and $L_{2-}$, norms of $\left|\varphi_{t}\right|^{p}$. But $\left\|\left|\varphi_{t}\right|^{p}\right\|_{1}=\left\|\varphi_{t}\right\|_{p}^{p}$ and $\left\|\left|\varphi_{t}\right|^{p}\right\|_{2}^{2}=\left\|\varphi_{t}\right\|_{2 p}^{2 p}$. Therefore one can use the induction proof on pp. 262-264 in [Rob], starting from the $L_{2}$-estimate (16), to deduce bounds on $\left\|S_{t}^{\rho}\right\|_{2 \rightarrow \infty}$. These bounds are the direct analogue of the bounds on p. 264 of [Rob],

$$
\left\|S_{t}^{\rho}\right\|_{2 \rightarrow \infty} \leqq a k\left(b \gamma^{2} \varepsilon \mu t / k\right)^{-k / 4} e^{-\lambda_{1} t} e^{\rho^{2}(1+\varepsilon) t+|\rho| v t} e^{\gamma^{2} \varepsilon \mu t},
$$

and are valid for all $t>0, \rho \in \mathbf{R}, \gamma \in\langle 0,1]$ and $\varepsilon \in\langle 0,1]$ with the values of $a$ and $b$ dependent only on the group, the basis $b_{1}, \ldots, b_{d}$ and the constant $\alpha$ in the Nash inequality Corollary 3.10 . Now if $\varepsilon \mu t \leqq 1$ set $\gamma=1$ and if $\varepsilon \mu t \geqq 1$ set $\gamma=(\varepsilon \mu t)^{-1 / 2}$. Then, with redefined values of $a$ and $\varepsilon$, one obtains bounds

$$
\left\|S_{t}^{\rho}\right\|_{2 \rightarrow \infty} \leqq a(1 \wedge \varepsilon \mu t)^{-k / 4} e^{-\lambda_{1} t} e^{\rho^{2}(1+\varepsilon) t+|\rho| v t}
$$

for all $t>0, \rho \in \mathbf{R}$ and $\varepsilon \in\langle 0,1]$. But by duality

$$
\left\|S_{t}^{\rho}\right\|_{1 \rightarrow \infty} \leqq\left\|S_{t / 2}^{\rho}\right\|_{1 \rightarrow 2}\left\|S_{t / 2}^{\rho}\right\|_{2 \rightarrow \infty}=\left\|S_{t / 2}^{-\rho}\right\|_{2 \rightarrow \infty}\left\|S_{t / 2}^{\rho}\right\|_{2 \rightarrow \infty} .
$$

Hence one obtains bounds

$$
\left\|S_{t}^{\rho}\right\|_{1 \rightarrow \infty} \leqq a(1 \wedge \varepsilon \mu t)^{-k / 2} e^{-\lambda_{1} t} e^{\rho^{2}(1+\varepsilon) t+|\rho| v t}
$$

for all $t>0, \rho \in \mathbf{R}$ and $\varepsilon \in\langle 0,1]$ and again a redefined value of $a$. Consequently

$$
\left|\kappa_{t}(x ; y)\right| \leqq a(1 \wedge \varepsilon \mu t)^{-k / 2} e^{-\lambda_{1} t} e^{\rho^{2}(1+\varepsilon) t+|\rho| v t+\rho(\psi(x)-\psi(y))}
$$


for all $t>0$ and $x, y \in \mathbf{R}^{k}$. The value of $a$ now depends on the group, the dimension $k$, the basis $b_{1}, \ldots, b_{d}$ and the constant $\alpha$ in the Nash inequality Corollary 3.10 , but is independent of the coefficients of $H$ and of $\varepsilon \in\langle 0,1]$. Minimizing over $\psi \in D_{C}$ one deduces that

$$
\left|\kappa_{t}(x ; y)\right| \leqq a(1 \wedge \varepsilon \mu t)^{-k / 2} e^{-\lambda_{1} t} e^{\rho^{2}(1+\varepsilon) t-|\rho| d_{U, C}(x ; y)+|\rho| v t} .
$$

This proves the first part of the theorem if the weak Malcev basis has the ideal property (3). We next remove this condition. By Lemma 2.2 there exists a weak Malcev basis $\tilde{a}_{1}, \ldots, \tilde{a}_{d_{\mathrm{m}}+k}$ passing through $m$ which has the ideal property. Let $c$ and $\sigma$ be as in Lemma 2.3 and let $\kappa_{t}$ and $\tilde{\kappa}_{t}$ be the two associated reduced kernels. Then it follows from the Gaussian bounds for $\tilde{\kappa}_{t}$ and Lemma 2.4 that

$$
\begin{aligned}
\left|\kappa_{t}(x ; y)\right| & =\left|c^{2} e^{i(\sigma(x)-\sigma(y))} \tilde{\kappa}_{t}(\theta(x) ; \theta(y))\right| \\
& \leqq c^{2} a(1 \wedge \varepsilon \mu t)^{-k / 2} e^{-\lambda_{1} t} e^{\rho^{2}(1+\varepsilon) t-|\rho| d_{\tilde{U}, C}(\theta(x), \theta(y))+|\rho| v t} .
\end{aligned}
$$

Hence it remains to prove that $d_{\tilde{U}, C}(\theta(x) ; \theta(y))=d_{U, C}(x ; y)$.

Now let $V$ be the unitary map as in Lemma 2.3. Further let $\psi \in C_{c}^{\infty}\left(\mathbf{R}^{k}\right)$ and set $\Psi=\psi \circ \theta$. Then $\left(V^{*} \Psi\right)(x)=c^{-1} e^{-l \sigma(x)} \psi(x)$. So

$$
\begin{aligned}
\left(\tilde{B}_{l} \Psi\right)\left(\theta^{-1}(x)\right) & =\left(V B_{l} V^{*} \Psi\right)\left(\theta^{-1}(x)\right)=c e^{i \sigma(x)}\left(B_{i} V^{*} \Psi\right)(x) \\
& =\left(B_{i} \psi\right)(x)-i\left(B_{l}^{\circ} \sigma\right)(x) \psi(x)
\end{aligned}
$$

for all $x \in \mathbf{R}^{k}$. Hence $\left(\tilde{B}_{i}^{\circ} \Psi\right)\left(\theta^{-1}(x)\right)=\left(B_{i}^{\circ} \psi\right)(x)$ and $\tilde{B}_{i}^{\circ} \Psi=\left(B_{i}^{\circ} \psi\right) \circ \theta$. From this identity one easily derives the transformation formula for the distances and the proof of the first part of the theorem is complete. The second part follows by minimizing over $\rho$.

There is another description of the distance $d_{U, C}$ which allows one to reformulate the statement of the theorem in a more geometric manner.

Each $B_{i}^{\circ}$ is a vector field on $\mathbf{R}^{k}$. But the algebra generated by the $B_{l}$ consists of all differential operators with polynomial coefficients, ([CoG] Theorem 4.1.1(i)), and the differential operator $\partial / \partial x_{j}$ has no constant term. It follows that the vector fields $B_{1}^{\circ}, \ldots, B_{d}^{\circ}$ generate the tangent space at any point of $\mathbf{R}^{k}$. We now define a geometric distance on $\mathbf{R}^{k}$ as in [NSW]. For $\delta>0$ let $C(\delta)$ be the set of all absolutely continuous functions $\gamma:[0,1] \rightarrow G$ which satisfy the differential equation

$$
\dot{\gamma}(t)=\left.\sum_{l=1}^{d} \gamma_{l}(t) B_{l}^{\circ}\right|_{\gamma(t)},
$$

almost everywhere, with

$$
\sum_{i, j=1}^{d}\left(C^{-1}\right)_{i j} \gamma_{i}(t) \gamma_{J}(t)<\delta^{2}
$$

for all $t \in[0,1]$, where $C$ denotes the matrix of coefficients. Then define the distance $d_{U, C}^{\mathrm{g}}(x ; y)$ between two elements $x, y \in \mathbf{R}^{k}$ by

$$
d_{U, C}^{\mathrm{g}}(x ; y)=\inf \left\{\delta>0: \exists_{\gamma \in C(\delta)}[\gamma(0)=x \text { and } \gamma(1)=y]\right\} .
$$


The distance $d_{U, C}^{\mathrm{g}}$ induces the Euclidean topology on $\mathbf{R}^{k}$.

Lemma 4.2. The distances $d_{U, C}$ and $d_{U, C}^{\mathrm{g}}$ are equal.

Proof. Let $x, y \in \mathbf{R}^{k}, \psi \in D_{C}, \delta>0$ and $\gamma \in C(\delta)$ with $\gamma(0)=x$ and $\gamma(1)=y$. Write

$$
\dot{\gamma}(t)=\left.\sum_{l=1}^{d} \gamma_{i}(t) B_{l}^{\circ}\right|_{\gamma(t)},
$$

with

$$
\sum_{i, j=1}^{d}\left(C^{-1}\right)_{l j} \gamma_{l}(t) \gamma_{J}(t)<\delta^{2}
$$

for almost every $t \in[0,1]$.

Now denote the inner product on $\mathbf{R}^{d}$ by $\langle\cdot, \cdot\rangle$, the norm by $|\cdot|$, set $\left[\left(B^{\circ} \psi\right)(z)\right]=\left(\left(B_{1}^{\circ} \psi\right)(z), \ldots,\left(B_{d}^{\circ} \psi\right)(z)\right) \in \mathbf{R}^{d}$ for all $z \in \mathbf{R}^{k}$ and $[\dot{\gamma}(t)]=\left(\gamma_{1}(t), \ldots\right.$, $\left.\gamma_{d}(t)\right)$. Then

$$
\begin{aligned}
|\psi(y)-\psi(x)| & =\left|\int_{0}^{1} d t \frac{d}{d t} \psi(\gamma(t))\right|=\left|\int_{0}^{1} d t \sum_{l=1}^{d} \gamma_{i}(t)\left(B_{i}^{\circ} \psi\right)(\gamma(t))\right| \\
& \leqq \int_{0}^{1} d t\left|\left\langle C^{-1 / 2}[\dot{\gamma}(t)], C^{1 / 2}\left[\left(B^{\circ} \psi\right)(\gamma(t))\right]\right\rangle\right| \\
& \leqq \int_{0}^{1} d t\left|C^{-1 / 2}[\dot{\gamma}(t)]\right|\left|C^{1 / 2}\left[\left(B^{\circ} \psi\right)(\gamma(t))\right]\right| \leqq \int_{0}^{1} d t \delta \cdot 1=\delta .
\end{aligned}
$$

Therefore $d_{U, C}(x ; y) \leqq d_{U, C}^{\mathrm{g}}(x ; y)$. by

Alternatively, fix $x_{0}, y_{0} \in \mathbf{R}^{k}$ and let $n=d_{U, C}^{\mathrm{g}}\left(x_{0} ; y_{0}\right)+1$. Define $\varphi_{n}: \mathbf{R} \rightarrow \mathbf{R}$

$$
\varphi_{n}(x)= \begin{cases}|x| & \text { if }|x| \leqq n \\ 2 n-|x| & \text { if } n<|x| \leqq 2 n \\ 0 & \text { if }|x|>2 n .\end{cases}
$$

Then $\varphi_{n} \in C_{c}(\mathbf{R})$ and $\left|\varphi_{n}(x)-\varphi_{n}(y)\right| \leqq|x-y|$ for all $x, y \in \mathbf{R}$. Define $\chi_{n}$ : $\mathbf{R}^{k} \rightarrow \mathbf{R}$ by $\chi_{n}(x)=\varphi_{n}\left(d_{U, C}^{\mathrm{g}}\left(x ; y_{0}\right)\right)$. Then $\chi_{n} \in C_{c}\left(\mathbf{R}^{k}\right)$ and $\left|\chi_{n}\left(x_{1}\right)-\chi_{n}\left(x_{2}\right)\right| \leqq$ $d_{U, C}^{\mathrm{g}}\left(x_{1} ; x_{2}\right)$ for all $x_{1}, x_{2} \in \mathbf{R}^{k}$.

Next we regularize $\chi_{n}$. Fix a positive $\tau \in C_{c}^{\infty}\left(\mathbf{R}^{k}\right)$ with integral equal to one. For $m \in \mathbf{N}$ define $\tau_{m} \in C_{c}^{\infty}\left(\mathbf{R}^{k}\right)$ by $\tau_{m}(x)=m^{k} \tau\left(m^{-1} x\right)$ and $\psi_{n m}: \mathbf{R}^{k} \rightarrow \mathbf{R}$ by

$$
\psi_{n m}=\tau_{m} * \chi_{n} \text {. }
$$

Here $*$ is the convolution on the commutative group $\mathbf{R}^{k}$. Then $\psi_{n m} \in C_{c}^{\infty}\left(\mathbf{R}^{k}\right)$. Moreover one has

$$
\lim _{m \rightarrow \infty} \psi_{n m}\left(x_{0}\right)=\varphi_{n}\left(d_{U, C}^{\mathrm{g}}\left(x_{0} ; y_{0}\right)\right)=d_{U, C}^{\mathrm{g}}\left(x_{0} ; y_{0}\right)
$$

For all $i \in\{1, \ldots, d\}, x \in \mathbf{R}^{k}$ and $t>0$ one has

$$
\left|\chi_{n}\left(\exp \left(-t B_{l}^{\circ}\right)(x)\right)-\chi_{n}(x)\right| \leqq d_{U, C}^{\mathrm{g}}\left(\exp \left(-t B_{i}^{\circ}\right)(x) ; x\right) \leqq\left(\left(C^{-1}\right)_{l i}\right)^{1 / 2} t .
$$


Since the representation $V$ on $L_{\infty}\left(\mathbf{R}^{k}\right)$ defined by $\left(V_{t} \omega\right)(x)=\omega\left(\exp \left(-t B_{i}^{\circ}\right)(x)\right)$ is weakly* continuous it follows that $\chi_{n}$ is in the domain of the operator $B_{i}^{\circ}$, viewed as an operator on $L_{\infty}\left(\mathbf{R}^{k}\right)$. Next we argue that $\sum_{l, j=1}^{d} c_{i j}\left(B_{i}^{\circ} \chi_{n}\right)(x)\left(B_{j}^{\circ} \chi_{n}\right)(x) \leqq 1$ for all $x \in \mathbf{R}^{k}$. One has for all $x \in \mathbf{R}^{k}$ :

$$
\begin{aligned}
& \left(\sum_{i, j=1}^{d} c_{i j}\left(B_{i}^{\circ} \chi_{n}\right)(x)\left(B_{j}^{\circ} \chi_{n}\right)(x)\right)^{1 / 2}=\left|C^{1 / 2}\left[\left(B^{\circ} \chi_{n}\right)(x)\right]\right| \\
& =\sup _{\substack{\xi \in \mathbf{R}^{d} \\
|\xi|=1}}\left\langle\xi, C^{1 / 2}\left[\left(B^{\circ} \chi_{n}\right)(x)\right]\right\rangle=\sup _{|\xi|=1} C^{1 / 2} \xi \cdot\left[\left(B^{\circ} \chi_{n}\right)(x)\right] .
\end{aligned}
$$

Now for all $t>0$ the path $\gamma(s)=\exp \left(s t C^{1 / 2} \xi \cdot B^{\circ}\right)(x)$ is a $C^{\infty}$-path from $x$ to $\exp \left(t C^{1 / 2} \xi \cdot B^{\circ}\right)(x)$ and $[\dot{\gamma}(s)]=t C^{1 / 2} \xi$ for all $s$. So

$$
\left\langle[\dot{\gamma}(s)], C^{-1}[\dot{\gamma}(s)]\right\rangle=\left\langle t C^{1 / 2} \xi, C^{-1} t C^{1 / 2} \xi\right\rangle=t^{2},
$$

and hence $\gamma \in C(t)$. Therefore $d_{U, C}^{\mathrm{g}}\left(\exp \left(t C^{1 / 2} \xi \cdot B^{\circ}\right)(x) ; x\right) \leqq t$ and $C^{1 / 2} \xi$. $\left[\left(B^{\circ} \chi_{n}\right)(x)\right] \leqq 1$ by an estimate as we used above for the proof that $\chi_{n}$ is differentiable.

Next, note that the representation $V$ leaves $C_{c}^{\infty}\left(\mathbf{R}^{k}\right)$ invariant. For all $f \in$ $L_{1}\left(\mathbf{R}^{k}\right)$ and $\omega \in C_{c}^{\infty}\left(\mathbf{R}^{k}\right)$ define

$$
F=\left\{\psi \in D\left(B_{l}^{\circ}\right):\left(f,\left(B_{i}^{\circ} \omega\right) * \psi\right)=\left(\tilde{\omega} * f, B_{i}^{\circ} \psi\right)\right\},
$$

where $\tilde{\omega}(x)=\omega(-x)$. Then $C_{c}^{\infty}\left(\mathbf{R}^{k}\right) \subset F$ and $F$ is weakly* closed in $D\left(B_{i}^{\circ}\right)$, so $F=D\left(B_{l}^{\circ}\right)$. Therefore

$$
B_{i}^{\circ}(\omega * \psi)=\left(B_{i}^{\circ} \omega\right) * \psi=\omega * B_{l}^{\circ} \psi
$$

for all $\psi \in D\left(B_{i}^{\circ}\right)$. In particular:

$$
B_{i}^{\circ} \psi_{n m}=\tau_{m} * B_{i}^{\circ} \chi_{n},
$$

and for all $x \in \mathbf{R}^{k}$ one obtains

$$
\begin{aligned}
& \left(\sum_{i, j=1}^{d} c_{i j}\left(B_{i}^{\circ} \psi_{n m}\right)(x)\left(B_{j}^{\circ} \psi_{n m}\right)(x)\right)^{1 / 2}=\left|C^{1 / 2}\left[\left(B^{\circ} \psi_{n m}\right)(x)\right]\right| \\
& \quad=\sup _{|\xi|=1}\left\langle\xi, C^{1 / 2}\left[\left(B^{\circ} \psi_{n m}\right)(x)\right]\right\rangle=\sup _{|\xi|=1}\left(\tau_{m} *\left\langle\xi, C^{1 / 2}\left[B^{\circ} \chi_{m}\right]\right\rangle\right)(x) \\
& \quad \leqq \sup _{|\xi|=1}\left\|\tau_{m}\right\|_{1}|\xi|||\left|C^{1 / 2}\left[B_{i}^{\circ} \chi_{m}\right]\right| \|_{\infty} \leqq 1 .
\end{aligned}
$$

Hence $\psi_{n m} \in D_{C}$ and $d_{U, C}^{\mathrm{g}}\left(x_{0} ; y_{0}\right) \leqq d_{U, C}\left(x_{0} ; y_{0}\right)$.

It follows from the general theory of strongly elliptic operators that the semigroup generated by a closed strongly elliptic operator with real principal coefficients is holomorphic in the open right half-plane. Then, by the discussion in Sect. 3, the corresponding reduced kernel extends to a function which is analytic in the halfplane. Therefore it is of interest to examine bounds on the kernel for complex $t$. This is particularly simple if there are no first-order terms, i.e., if $c_{i}=0$. In this case the bounds of Theorem 4.1 give

$$
\left|\kappa_{t}(x ; y)\right| \leqq a(1 \wedge \varepsilon \mu t)^{-k / 2} e^{-\lambda_{1} t} \exp \left(-d_{U, C}(x ; y)^{2}(4(1+\varepsilon) t)^{-1}\right)
$$

for all $t>0$ and $\varepsilon \in\langle 0,1]$. These bounds have the following analogue. 
Corollary 4.3. Let $l \in \mathrm{g}^{*}, a_{1}, \ldots, a_{d_{\mathrm{m}}}, \ldots, a_{d_{\mathrm{m}}+k}$ be a weak Malcev basis passing through a polarizing subalgebra $\mathrm{m}$ of $l$ and $U$ the corresponding basis realization in $L_{2}\left(\mathbf{R}^{k}\right)$. Let $H$ be a pure second-order operator associated with the real, symmetric, strictly positive-definite matrix $C=\left(c_{i j}\right)$, and the basis $b_{1}, \ldots, b_{d}$ of g. Further let $\kappa$ denote the corresponding reduced kernel. Then there exists an $a>0$, independent of the coefficients $C$, such that

$$
\begin{aligned}
\left|\kappa_{z}(x ; y)\right| \leqq & a(\varepsilon \cos \theta)^{-k / 2}(1 \wedge \varepsilon \mu \operatorname{Re} z)^{-k / 2} e^{-\lambda_{1} \operatorname{Re} z} \\
& \times \exp \left(-d_{U, C}(x ; y)^{2} \operatorname{Re}(4(1+\varepsilon) z)^{-1}\right)
\end{aligned}
$$

for all $z \in \mathbf{C}$ with $\operatorname{Re} z>0$ and all $\varepsilon \in\langle 0,1]$, where $\theta=\arg z$.

Proof. We adapt the general reasoning of Davies [Dav2], Lemma 3.4.6 and Theorem 3.4.8.

First remark that if $z=t+i s$ then

$$
\left\|S_{z}\right\|_{1 \rightarrow 2}=\left\|S_{t}\right\|_{1 \rightarrow 2}=\left\|S_{t}\right\|_{2 \rightarrow \infty}=\left\|S_{\bar{z}}\right\|_{2 \rightarrow \infty}
$$

because $H$ is self-adjoint on $L_{2}\left(\mathbf{R}^{k}\right)$. Therefore

$$
\left\|S_{z}\right\|_{1 \rightarrow \infty} \leqq\left\|S_{z / 2}\right\|_{1 \rightarrow 2}\left\|S_{z / 2}\right\|_{2 \rightarrow \infty}=\left(\left\|S_{t / 2}\right\|_{2 \rightarrow \infty}\right)^{2} .
$$

Hence, by (20) with $\rho=0$ and $\varepsilon=1$, one has bounds

$$
\left|\kappa_{z}(x ; y)\right| \leqq\left\|S_{z}\right\|_{1 \rightarrow \infty} \leqq a(1 \wedge \mu t)^{-k / 2} e^{-\lambda_{1} t},
$$

with a redefined value of $a$, for all $z \in \mathbf{C}$ with $t=\operatorname{Re} z>0$. Then since $(1 \wedge t) \geqq$ $\left(1-e^{-t}\right)$ this gives

$$
\left|\kappa_{z}(x ; y)\right| \leqq a\left(1-e^{-\mu t}\right)^{-k / 2} e^{-\lambda_{1} t} .
$$

Alternatively, one can rephrase the bounds of Theorem 4.1 as

$$
\left|\kappa_{t}(x ; y)\right| \leqq a\left(1-e^{-\varepsilon \mu t}\right)^{-k / 2} e^{-\lambda_{1} t} e^{-d_{U, C}(x ; y)^{2}(4(1+\varepsilon) t)^{-1}},
$$

uniformly for all $t>0$ and $\varepsilon \in\langle 0,1]$.

Next for fixed $x, y \in \mathbf{R}^{k}, \varepsilon \in\langle 0,1]$ and $\varphi \in\langle 0, \pi / 2\rangle$ define the analytic function $F$ in the open right half-plane by

$$
F(z)=\kappa_{z^{-1}}(x ; y) e^{\lambda_{1} z^{-1}}\left(1-e^{-\varepsilon \mu z^{-1}}\right)^{k / 2} e^{b_{\varphi} e^{\ell(\pi / 2-\varphi)} d_{U, C}(x ; y)^{2} z},
$$

where $b_{\varphi}=(4(1+\varepsilon) \sin \varphi)^{-1}$. Then

$$
|F(t)| \leqq a
$$

for all $t>0$. Now it follows from a Duhamel estimate that

$$
\begin{aligned}
\left|1-e^{-s e^{-l \varphi}}\right| & \leqq\left|s e^{-l \varphi}\right| \int_{0}^{1} d \lambda\left|e^{-\lambda s e^{-l \varphi}}\right|=s \int_{0}^{1} d \lambda e^{-\lambda s \cos \varphi} \\
& =(\cos \varphi)^{-1}\left(1-e^{-s \cos \varphi}\right)=(\cos \varphi)^{-1}\left(1-e^{-\operatorname{Re} s e^{-l \varphi}}\right)
\end{aligned}
$$

for all $s>0$. Hence

$$
\begin{aligned}
\left|F\left(t e^{l \varphi}\right)\right| & \leqq a\left(1-e^{-\mu \operatorname{Re} t^{-1} e^{-i \varphi}}\right)^{-k / 2}\left|1-e^{-\varepsilon \mu t^{-1} e^{-l \varphi}}\right|^{k / 2} \\
& \leqq a\left(1-e^{-\varepsilon \mu \operatorname{Re} t^{-1} e^{-l \varphi}}\right)^{-k / 2}\left|1-e^{-\varepsilon \mu t^{-1} e^{-i \varphi}}\right|^{k / 2} \\
& \leqq a(\cos \varphi)^{-k / 2} .
\end{aligned}
$$


Moreover, if $\theta \in[0, \varphi]$ then

$$
\left|F\left(t e^{i \theta}\right)\right| \leqq a(\cos \theta)^{-k / 2} e^{b_{\varphi} d_{U, C}(x ; y)^{2} t \sin (\varphi-\theta)} \leqq a(\cos \varphi)^{-k / 2} e^{b_{\varphi} d_{U, C}(x ; y)^{2} t \sin \varphi} .
$$

Therefore the Phragmén-Lindelöf theorem implies that

$$
|F(z)| \leqq c(\cos \varphi)^{-k / 2}
$$

for all $z$ with $\arg z \in[0, \varphi]$, for a suitable $c>0$, depending only on $a$. Similar reasoning leads to an identical bound for $z$ with $\arg z \in[-\varphi, 0]$. But since

$$
\kappa_{z}(x ; y)=F\left(z^{-1}\right)\left(1-e^{-\varepsilon \mu z}\right)^{-k / 2} e^{-\lambda_{1} z} e^{-b_{\varphi} z^{-1} e^{\imath(\pi / 2-\varphi)} d_{U, C}(x ; y)^{2}},
$$

one concludes that

$$
\left|\kappa_{z}(x ; y)\right| \leqq c(\cos \varphi)^{-k / 2}\left|1-e^{-\varepsilon \mu z}\right|^{-k / 2} e^{-\lambda_{1} \operatorname{Re} z} e^{-b_{\varphi} d_{U, C}(x, y)^{2} t^{-1} \sin (\varphi-|\theta|)}
$$

for all $z=t e^{\imath \theta} \in \mathbf{C}$ with $|\arg z| \leqq \varphi$. Now, however, $1-e^{-\operatorname{Re} z} \leqq\left|1-e^{-z}\right|$ for all $z \in \mathbf{C}$ with $\operatorname{Re} z>0$, by the triangle inequality. In addition $1 \wedge t \leqq$ $\left(1-e^{-1}\right)^{-1}\left(1-e^{-t}\right)$ for all $t>0$. Therefore

$$
\left|\kappa_{z}(x ; y)\right| \leqq c(\cos \varphi)^{-k / 2}(1 \wedge \varepsilon \mu \operatorname{Re} z)^{-k / 2} e^{-\lambda_{1} \operatorname{Re} z} e^{-b_{\varphi} d_{U, C}(x, y)^{2} t^{-1} \sin (\varphi-|\theta|)}
$$

for all $z=t e^{l \theta} \in \mathbf{C}$ with $|\arg z| \leqq \varphi$.

Next for $z \in \mathbf{C}$ with $\operatorname{Re} z>0$ and $\operatorname{Im} z \neq 0$ choose $\varphi \in\langle 0, \pi / 2\rangle$ such that $\varepsilon \tan \varphi=\tan |\theta|$. Then $\sin (\varphi-|\theta|)(\sin \varphi)^{-1}=(1-\varepsilon) \cos \theta$ and $\cos \varphi=\varepsilon\left(\varepsilon^{2}+\right.$ $\left.\tan ^{2} \theta\right)^{-1 / 2} \geqq \varepsilon \cos \theta$, so

$$
\left|\kappa_{z}(x ; y)\right| \leqq c(\varepsilon \cos \theta)^{-k / 2}(1 \wedge \varepsilon \mu \operatorname{Re} z)^{-k / 2} e^{-\lambda_{1} \operatorname{Re} z} e^{-(1-\varepsilon) d_{U, C}(x ; y)^{2} \operatorname{Re}(4(1+\varepsilon) z)^{-1}} .
$$

Finally, set $\delta=2 \varepsilon(1-\varepsilon)^{-1}$ so that $(1+\varepsilon)(1-\varepsilon)^{-1}=(1+\delta)$. Then $\varepsilon=\delta(2+$ $\delta)^{-1} \geqq \delta / 3$ for $\delta \in\langle 0,1]$ and

$$
\left|\kappa_{z}(x ; y)\right| \leqq c 3^{k}(\delta \cos \theta)^{-k / 2}(1 \wedge \delta \mu \operatorname{Re} z)^{-k / 2} e^{-\lambda_{1} \operatorname{Re} z} e^{-d_{U, C}(x ; y)^{2} \operatorname{Re}(4(1+\delta) z)^{-1}}
$$

for all $z \in \mathbf{C}$ with $\operatorname{Re} z>0$ and $\theta=\arg z$ and for all $\delta \in\langle 0,1]$. Thus the statement of the corollary is established by a change of notation.

The estimates of Theorem 4.1 depend critically on the reality of the principal coefficients $\left(c_{l j}\right)$ but less critically on the reality of the first-order coefficients $c_{l}$. One can adapt the foregoing arguments to bound the reduced kernels associated with second-order operators with complex-valued $c_{i}$ at the cost of forfeiting control over the large $t$ behaviour.

Corollary 4.4. Let $l \in \mathfrak{g}^{*}, a_{1}, \ldots, a_{d_{11}}, \ldots, a_{d_{11}+k}$ be a weak Malcev basis passing through a polarizing subalgebra $m$ of $l$ and $U$ the corresponding basis realization in $L_{2}\left(\mathbf{R}^{k}\right)$. Let $H$ be a second-order operator associated with the real symmetric matrix of principal coefficients $C=\left(c_{l J}\right)$, the first-order coefficients $c_{l} \in \mathbf{C}$ and the basis $b_{1}, \ldots, b_{d}$ of $\mathfrak{g}$. Further let $\kappa_{t}$ denote the corresponding reduced kernel. Then for all $\varepsilon \in\langle 0,1]$ there exists an $a_{\varepsilon}>0$ and $\omega_{\varepsilon} \geqq 0$ such that

$$
\left|\kappa_{t}(x ; y)\right| \leqq a_{\varepsilon} t^{-k / 2} e^{\left(\omega_{\epsilon} t\right.} e^{-d_{U, C}(x ; y)^{2}(4(1+\varepsilon) t)^{-1}}
$$

uniformly for all $t>0$ and $x, y \in \mathbf{R}^{k}$. 
Proof. The proof is an elaboration of the proof of Theorem 4.1. We briefly comment on the extra features.

First, in the calculation of $d\left\|\varphi_{t}\right\|_{2}^{2} / d t$ one has additional terms

$$
X_{1}=2 \operatorname{Re} \sum_{i=1}^{d} c_{i}\left(\varphi_{t},\left(B_{l}+\rho \psi_{l}\right) \varphi_{t}\right)
$$

But these can be handled by $\left(\varepsilon, \varepsilon^{-1}\right)$-estimates. For example, one readily finds that

$$
\begin{aligned}
\left|X_{1}\right| & \leqq \varepsilon \mu \sum_{i=1}^{d}\left\|B_{i} \varphi_{t}\right\|_{2}^{2}+\varepsilon^{-1} v^{2}\left\|\varphi_{t}\right\|_{2}^{2}+2|\rho| v\left\|\varphi_{t}\right\|_{2}^{2} \\
& \leqq \varepsilon\left(\varphi_{t}, H_{0} \varphi_{t}\right)+\left(\varepsilon^{-1} v^{2}+2|\rho| v\right)\left\|\varphi_{t}\right\|_{2}^{2} .
\end{aligned}
$$

Therefore choosing $\varepsilon=2$ and using the previous estimates one finds that

$$
\frac{d}{d t}\left\|\varphi_{t}\right\|_{2}^{2} \leqq 2(|\rho|+v / 2)^{2}\left\|\varphi_{t}\right\|_{2}^{2}
$$

and then, by integration,

$$
\left\|S_{t}^{\rho}\right\|_{2 \rightarrow 2} \leqq e^{(|\rho|+v / 2)^{2} t}
$$

for all $t>0$ and $\rho \in \mathbf{R}$.

Similar modifications are necessary for the estimation of $d\left\|\varphi_{t}\right\|_{2 p}^{2 p} / d t$. Now one has additional terms

$$
\begin{aligned}
W_{1} & =-2 p \operatorname{Re} \sum_{i=1}^{d} c_{i}\left(\varphi_{t}^{p} \overline{\varphi_{t}}{ }^{p-1},\left(B_{l}+\rho \psi_{l}\right) \varphi_{t}\right) \\
& =-2 p \operatorname{Re} \sum_{l=1}^{d} c_{l}\left(\left|\varphi_{t}\right|^{p-1} \varphi_{t},\left|\varphi_{t}\right|^{p-1} B_{l} \varphi_{t}\right)-2 p \rho \operatorname{Re} \sum_{l=1}^{d} c_{l}\left(\left|\varphi_{t}\right|^{p}, \psi_{i}\left|\varphi_{t}\right|^{p}\right) .
\end{aligned}
$$

Hence

$$
\begin{aligned}
\left|W_{1}\right| & \leqq \varepsilon p v \sum_{l, j=1}^{d} c_{l j}\left(\left|\varphi_{t}\right|^{p-1} B_{l} \varphi_{t},\left|\varphi_{t}\right|^{p-1} B_{j} \varphi_{t}\right)+\varepsilon^{-1} p v\left\|\varphi_{t}^{p}\right\|_{2}^{2}+2 p|\rho| v\left\|\varphi_{t}^{p}\right\|_{2}^{2} \\
& =p \sum_{i, j=1}^{d} c_{i j}\left(\left|\varphi_{t}\right|^{p-1} B_{i} \varphi_{t},\left|\varphi_{t}\right|^{p-1} B_{J} \varphi_{t}\right)+\left(p v^{2}+2 p|\rho| v\right)\left\|\varphi_{t}^{p}\right\|_{2}^{2}
\end{aligned}
$$

if one chooses $\varepsilon=v^{-1}$. Finally, one obtains a differential inequality which differs from the earlier one for pure second-order operators only in the terms proportional to $\left\|\varphi_{t}\right\|_{2 p}$. Now one deduces that

$$
\frac{d}{d t}\left\|\varphi_{t}\right\|_{2 p}^{2 p} \leqq-\mu \sum_{l=1}^{d}\left\|B_{i}^{\circ}\left|\varphi_{t}\right|^{p}\right\|_{2}^{2}+\left(4 p^{2} \rho^{2}+2 p|\rho| v+p v^{2}\right)\left\|\varphi_{t}\right\|_{2}^{2},
$$

instead of the inequality (18) and

$$
\frac{d}{d t}\left\|\varphi_{t}\right\|_{2 p} \leqq-\mu(2 p)^{-1}\left\|\varphi_{t}\right\|_{2 p}^{1-2 p} \sum_{i=1}^{d}\left\|B_{i}^{\circ}\left|\varphi_{t}\right|^{p}\right\|_{2}^{2}+\left(2 p \rho^{2}+|\rho| v+v^{2} / 2\right)\left\|\varphi_{t}\right\|_{2 p}
$$


which is the direct analogue of (19). The coefficient $2 p \rho^{2}$ is replaced by $2 p \rho^{2}+$ $|\rho| v+v^{2} / 2$. Hence the bounds on the reduced kernel become

$$
\begin{aligned}
\left|\kappa_{t}(x ; y)\right| & \leqq a_{\varepsilon} t^{-k / 2} e^{\left(\rho^{2}+\rho v\right)(1+\varepsilon) t-\rho d_{U, C}(x ; y)} e^{4^{-1} v^{2}(1+\varepsilon) t} \\
& \leqq a_{\varepsilon} t^{-k / 2} e^{\rho^{2}(1+2 \varepsilon) t-\rho d_{U, C}(x ; y)} e^{w^{2}\left(1+\varepsilon^{-1}(1+\varepsilon)^{2}\right) t}
\end{aligned}
$$

uniformly for all $\rho, t, \varepsilon>0$ where $w=v / 2$. Hence minimizing over $\rho$, replacing $2 \varepsilon$ by $\varepsilon$ and redefining $a_{\varepsilon}$ gives the desired bounds.

There is also an analogue of Corollary 4.5 for operators with real principal coefficients and purely imaginary first-order coefficients. The resulting $H$ is still self-adjoint on $L_{2}\left(\mathbf{R}^{k}\right)$ and hence one has bounds

$$
\left\|S_{z}\right\|_{1 \rightarrow \infty} \leqq\left\|S_{z / 2}\right\|_{1 \rightarrow 2}\left\|S_{z / 2}\right\|_{2 \rightarrow \infty}=\left(\left\|S_{t / 2}\right\|_{2 \rightarrow \infty}\right)^{2}
$$

for all $z \in \mathbf{C}$ with $t=\operatorname{Re} z>0$. Thus it follows from Corollary 4.4 that one has bounds

$$
\left|\kappa_{z}(x ; y)\right| \leqq a t^{-k / 2} e^{\omega t}
$$

for all $z \in \mathbf{C}$ with $t=\operatorname{Re} z>0$ uniformly for $x, y \in \mathbf{R}^{k}$. Now the arguments of Davies [Dav2], Sect. 3.4, apply directly to give the analogue of Corollary 4.3.

Corollary 4.5. Let $l \in \mathfrak{g}^{*}, a_{1}, \ldots, a_{d_{11}}, \ldots, a_{d_{11}+k}$ be a weak Malcev basis passing through a polarizing subalgebra $m$ of $l$ and $U$ the corresponding basis realization in $L_{2}\left(\mathbf{R}^{k}\right)$. Let $H$ be a second-order operator associated with the real, symmetric, strictly positive-definite matrix $C=\left(c_{l j}\right)$, the imaginary first-order coefficients $c_{i}$ and the basis $b_{1}, \ldots, b_{d}$ of $\mathfrak{g}$. Further let $\kappa_{t}$ denote the corresponding reduced kernel. Then for all $\varepsilon \in\langle 0,1]$, there exists an $a_{\varepsilon}>0$, independent of the coefficients $C$, and an $\omega_{\varepsilon} \geqq 0$ such that

$$
\left|\kappa_{z}(x ; y)\right| \leqq a_{\varepsilon}(\cos \theta)^{-k / 2}(\operatorname{Re} z)^{-k / 2} e^{\omega_{\varepsilon} \operatorname{Re} z} e^{-d_{U, C}(x ; y)^{2} \operatorname{Re}(4(1+\varepsilon) z)^{-1}}
$$

for all $z \in \mathbf{C}$ with $\operatorname{Re} z>0$, where $\theta=\arg z$.

Finally we note that for strongly elliptic operators of order $m>2$ the method of this section does not work. The first problem is that there is no description of higher order strongly elliptic operators in terms of positivity of a matrix of principal coefficients. This can be bypassed by using the method of Sect. III.4 in [Rob]. But then one encounters $m^{\text {th }}$ order derivatives on the functions $\psi$ used in the perturbation argument. One could define inductively $D_{1}=D_{U, C}$ and

$$
D_{n}=\left\{\psi \in D_{n-1}: B_{i}^{\circ} \psi \in D_{n-1} \quad \text { for all } i \in\{1, \ldots, d\}\right\}
$$

for all $n \geqq 2$ and

$$
d_{n}(x ; y)=\sup _{\psi \in D_{n}}|\psi(x)-\psi(y)|
$$

Then it is readily verified that $d_{n}$ is non-degenerate and is a distance on $\mathbf{R}^{k}$. One can then obtain Gaussian type bounds for the reduced kernel of the semigroup generated by an $m^{\text {th }}$ order operator with the distance on $\mathbf{R}^{k}$ equal to $d_{m}$. In the situation of Sect. III.4 of [Rob] the corresponding distances $d_{1}, d_{2}, \ldots$ are all equivalent (see pp. 200-203), but in the present setting with the irreducible unitary representations the distance $d_{m}$ is not equivalent to $d_{1}=d_{U, C}=d_{U, C}^{\mathrm{g}}$ if $m$ is large, in general. 
One can prove bounds on the reduced kernels corresponding to $m^{\text {th }}$ order operators by exploiting the Nash inequalities Corollary 3.7 as in [Rob] Chapter III and one obtains that

$$
\left\|\kappa_{t}\right\|_{\infty}=\left\|S_{t}\right\|_{1 \rightarrow \infty} \leqq a t^{-k / m} e^{\omega t}
$$

for some $a>0$ and $\omega \in \mathbf{R}$, valid for all $t>0$. If the strongly elliptic operator is self-adjoint, with smallest eigenvalue $\lambda_{1}$ then $\left\|S_{t}\right\|_{2 \rightarrow 2} \leqq e^{-\lambda_{1} t}$ by spectral theory. So using the decomposition $S_{t}=S_{1} \circ S_{t-2} \circ S_{1}: L_{1} \rightarrow L_{2} \rightarrow L_{2} \rightarrow L_{\infty}$ one deduces that

$$
\left\|\kappa_{t}\right\|_{\infty}=\left\|S_{t}\right\|_{1 \rightarrow \infty} \leqq a(1 \wedge t)^{-k / m} e^{-\lambda_{1} t}
$$

for some $a>0$, valid for all $t>0$.

The same situation occurs if one attempts to derive Gaussian bounds for the higher order derivatives of the reduced kernel, even for second order operators. We are only able to derive Gaussian bounds in terms of the distance $d_{U, C}$ for the firstorder derivatives of the reduced kernels of semigroups generated by second-order operators:

$$
\left|\left(B_{i} \kappa_{t}\right)(x ; y)\right| \leqq a t^{-(k+1) / 2} e^{-b d_{U, C}(x ; y)^{2} t^{-1}}
$$

uniformly for all $i \in\{1, \ldots, k\}, t \in\langle 0,1]$ and $x, y \in \mathbf{R}^{k}$. Since we are not able to prove higher order kernel bounds with the distance $d_{U, C}$ we omit the proof.

\section{Kernel Bounds: Large $t$}

In this section we use spectral theory in combination with embedding arguments to establish bounds on the reduced kernel $\kappa_{t}$ associated with the semigroup $S$ generated by an $m^{\text {th }}$ order, formally self-adjoint operator. The arguments apply equally well to strongly elliptic operators or subelliptic operators. Self-adjointness is the important characteristic. There are two main features of these bounds. First, they still give the optimal decrease, $\exp \left(-\lambda_{1} t\right)$, as a function of $t$. Secondly, they establish that the kernel is "exponentially" decreasing on the diagonal. The earlier bounds did not give any estimate on the decrease of the kernel along the diagonal.

Let $U$ be the basis realization of the nilpotent Lie group $G$ corresponding to a weak Malcev basis $a_{1}, \ldots, a_{d_{\mathfrak{m}}}, \ldots, a_{d_{\mathfrak{m}}+k}$ passing through a polarizing subalgebra $\mathrm{m}$ for an $l \in \mathfrak{g}^{*}$ and let $C$ be a strongly elliptic, formally self-adjoint, $m^{\text {th }}$ order form. Set $H=d U(C)$ and let $\kappa$ be the corresponding reduced kernel. It follows from the general theory of elliptic operators that $H$ is self-adjoint on $L_{2}\left(\mathbf{R}^{k}\right)$. Moreover, it follows from Kirillov theory that the kernel $\kappa_{t}$ belongs to the Schwartz space $\mathscr{S}\left(\mathbf{R}^{k} \times \mathbf{R}^{k}\right)$. Therefore the self-adjoint semigroup $S$ generated by $H$ is trace class and $H$ has compact resolvent (see Theorem 2.5). Now we exploit these spectral properties to derive bounds on $\kappa_{t}$.

Since $\kappa_{t}$ belongs to the Schwartz space $\mathscr{S}\left(\mathbf{R}^{k} \times \mathbf{R}^{k}\right)$ it is polynomial decreasing, together with all its derivatives. But more is true, the kernel is "exponentially" decreasing.

Theorem 5.1. Let $U$ be the basis realization in $L_{2}\left(\mathbf{R}^{k}\right)$ of the nilpotent Lie group corresponding to $l \in \mathrm{g}^{*}$ and a weak Malcev basis passing through a polarizing subalgebra $\mathrm{m}$ of $l$. Further, let $\kappa$ be the reduced semigroup kernel corresponding to a self-adjoint, $m^{\text {th }}$ order, strongly elliptic operator $H$. Then there exist $\alpha \in[2, \infty\rangle$ 
and $b>0$ such that for all $z \in \mathbf{C}$ with $t=\operatorname{Re} z>0$ and all multi-indices $\beta$ and $\gamma$ there exists $c_{\beta, \gamma, t}>0$ such that

$$
\left|\left(D_{x}^{\beta} D_{y}^{\gamma} \kappa_{z}\right)(x ; y)\right| \leqq c_{\beta, \gamma, t} e^{-\lambda_{1} t} e^{-b|x|^{1 / \alpha}-b|y|^{1 / x}}
$$

uniformly for all $x, y \in \mathbf{R}^{k}$, where $\lambda_{1}$ denotes the smallest eigenvalue of the operator $H$. Moreover, the constants $c_{\beta, \gamma, t}$ can be chosen such that

for all $\beta$ and $\gamma$.

$$
\sup _{t \geqq 1} c_{\beta, \gamma, t}<\infty
$$

Proof. Let $\lambda_{1} \leqq \lambda_{2} \leqq \ldots$ denote the eigenvalues of the operator $H$, repeated according to multiplicity and let $\varphi_{1}, \varphi_{2}, \ldots$ be a corresponding orthonormal basis of eigenfunctions. Then $\varphi_{j} \in D^{\infty}(H)=\mathscr{S}\left(\mathbf{R}^{k}\right)$ for all $j$. We obtain bounds on $\kappa_{t}$ by examining the spectral decomposition

$$
\kappa_{z}(x ; y)=\sum_{j=1}^{\infty} e^{-\lambda_{j} z} \varphi_{j}(x) \overline{\varphi_{j}(y)}
$$

of the semigroup $S_{z}$ generated by $H$. This series converges in the $L_{2}$-sense, by general theory, but we will establish that the convergence is uniform. The estimates we obtain will even demonstrate that it converges in the $L_{p}$-sense for all $p \in[1, \infty]$.

Let $P_{j}$ and $Q_{j}, j \in\{1, \ldots, k\}$, be the self-adjoint operators on $L_{2}\left(\mathbf{R}^{k}\right)$ such that $\left(P_{J} f\right)(x)=i \partial_{j} f(x)$ and $\left(Q_{j} f\right)(x)=x_{j} f(x)$ for all $f \in \mathscr{S}\left(\mathbf{R}^{k}\right)$ and $x \in \mathbf{R}^{k}$. There exists, by [CoG] Theorem 4.1.1, an $n \in \mathbf{N}$ such that each $P_{j}$ and $Q_{j}$ is a linear combination of monomials of order at most $n$ in the $B_{i}$ on the Schwartz space. Hence, by [Rob] Corollary I.6.7, there exists $c \geqq 1$ such that $D\left(H^{n}\right) \subseteq D\left(H_{0}\right)$ and

$$
\left\|H_{0} \varphi\right\|_{2}^{2} \leqq c\left(\left\|H^{n} \varphi\right\|_{2}^{2}+\|\varphi\|_{2}^{2}\right)
$$

for all $\varphi \in D\left(H^{n}\right)$, where

$$
H_{0}=\sum_{j=1}^{k} P_{j}^{2}+Q_{j}^{2} .
$$

So $H_{0}^{2} \leqq c\left(H^{2 n}+I\right)$. Let $N(\lambda)$ and $N_{0}(\lambda)$ denote the number of eigenvalues of $H$ and $H_{0}$ which are less than or equal to $\lambda$, counted according to their multiplicity. Then it follows from the minimax theorem that

$$
N(\lambda) \leqq N_{0}\left(\left(c\left(\lambda^{2 n}+1\right)\right)^{1 / 2}\right) \leqq N_{0}\left(2 c \lambda^{n}\right)
$$

for all $\lambda \geqq \max \left(\left|\lambda_{1}\right|, 1\right)$. One can easily estimate $N_{0}$ and one has $N_{0}(\lambda) \leqq((\lambda-$ $1) / 2)^{k}$ for all $\lambda \geqq 1$. So $N(\lambda) \leqq c^{k} \lambda^{k n}$ if $\lambda \geqq \max \left(\left|\lambda_{1}\right|, 1\right)$. Then $j \leqq N\left(\lambda_{j}\right) \leqq$ $c^{k} \lambda_{j}^{k n}$ and hence

$$
\lambda_{j} \geqq\left(c^{-k} j\right)^{1 /(k n)}
$$

for all $j \in \mathbf{N}$ with $\lambda_{j} \geqq \max \left(\left|\lambda_{1}\right|, 1\right)$.

Alternatively, there exists $c>0$ such that $c\|H \varphi\| \leqq\left\|H_{0}^{m r} \varphi\right\|$ for all $\varphi \in$ $D\left(H_{0}^{m r}\right)$, where $r$ is the rank of the Lie algebra $\mathfrak{g}$. Then

$$
N(\lambda) \geqq N_{0}\left(c^{1 /(m r)} \lambda^{1 /(m r)}\right) \geqq 2^{-1}\left(c^{1 /(m r)} \lambda^{1 /(m r)}-k\right) \geqq c^{\prime} \lambda^{1 /(m r)}
$$


for all $\lambda$ sufficiently large. Hence

$$
\left|\lambda_{j}\right| \leqq b j^{m r}
$$

for some $b>0$, first for all sufficiently large $j$, but then by increasing $b$, if necessary, for all $j \in \mathbf{N}$.

Next we consider bounds on the eigenfunctions $\varphi_{j}$. If $T_{1}, \ldots, T_{q}$ are operators in $L_{2}\left(\mathbf{R}^{k}\right)$ and $\lambda>0$ then we define the Gevrey space $G_{\lambda}\left(T_{1}, \ldots, T_{q}\right)$ by

$$
G_{\lambda}\left(T_{1}, \ldots, T_{q}\right)=\bigcup_{s>0} G_{\lambda ; s}\left(T_{1}, \ldots, T_{q}\right),
$$

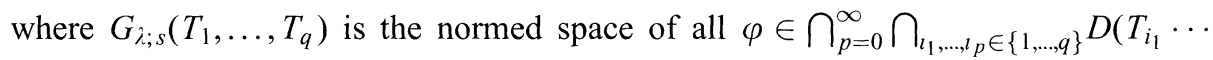
$T_{i_{p}}$ ) such that

$$
\sup _{p \in \mathbf{N}_{0}} \sup _{l_{1}, \ldots, i_{p} \in\{1, \ldots, q\}}\left(s^{p} p !^{\lambda}\right)^{-1}\left\|T_{i_{1}} \cdots T_{l_{p}} \varphi\right\|<\infty .
$$

Using the eigenvalue estimates one deduces

$$
\begin{aligned}
\left\|H^{p} \varphi_{j}\right\|_{2}=|\lambda,|^{p} & \leqq\left(b j^{m r}\right)^{p}=b^{p}\left(\left(j^{1 /(2 k n)}\right)^{p}\right)^{2 k m n r} \\
& \leqq b^{p}\left(e^{J^{1 /(2 k n)}} p !\right)^{2 k m n r}=e^{2 k m n r j^{1 /(2 k n)}} b^{p} p !^{2 k m n r}
\end{aligned}
$$

uniformly for all $p \in \mathbf{N}_{0}$ and $j \in \mathbf{N}$, so $\varphi_{j} \in G_{2 k m n r, b}(H)$, with norm bounded by $e^{2 k m n r j^{1 /(2 k n)}}$. It then follows from [E1R], Theorem 6.1, that

$$
G_{2 k m n r}(H)=G_{2 k n r}\left(B_{1}, \ldots, B_{d}\right) \subseteq G_{2 k n^{2} r}\left(P_{1}, \ldots, P_{k}, Q_{1}, \ldots, Q_{k}\right)=S_{\alpha, \ldots, \alpha}^{\alpha, \ldots, \alpha},
$$

where $\alpha=2 k n^{2} r$ and $S_{\alpha, \ldots, \alpha}^{\alpha, \ldots, \alpha}$ denotes the Gel'fand-Shilov space on $\mathbf{R}^{k}$ (see [GeS] Chapter IV). Now each function $\varphi \in S_{\alpha, \ldots, \alpha}^{\alpha, \ldots, \alpha}$ is infinitely differentiable and there exists $b^{\prime}>0$ (depending on $\varphi$ ) such that for every multi-index $\beta$ there exists $c^{\prime}>0$ such that

$$
\left|\left(D^{\beta} \varphi\right)(x)\right| \leqq c^{\prime} e^{-b^{\prime}|x|^{1 / x}}
$$

uniformly for all $x \in \mathbf{R}^{k}$. So

$$
\left|\left(D^{\beta} \varphi_{j}\right)(x)\right| \leqq c_{\beta, J} e^{-b,|x|^{1 / \alpha}}
$$

for some constants $b_{J}, c_{\beta, J}>0$. But if one traces the various constants then it follows that $b_{j}$ depends only on $b$ since each $\varphi_{j} \in G_{r ; b}(H)$ and $c_{\beta, j}$ can be estimated by a function which depends linearly on the norm of $\varphi_{j}$ in the space $G_{r ; b}(H)$. So $c_{\beta, J} \leqq c_{\beta} e^{2 k m n r j^{1 /(2 k n)}}$ for some $c_{\beta}$, independent of $j$. Thus

$$
\left|\left(D^{\beta} \varphi_{J}\right)(x)\right| \leqq c_{\beta} e^{2 k m n r j^{1 /(2 k n)}} e^{-b_{0}|x|^{1 / x}}
$$

for some constant $b_{0}>0$, uniformly for all multi-indices $\beta$, all $j \in \mathbf{N}$ and $x \in \mathbf{R}^{k}$.

It now easily follows that for all multi-indices $\beta, \gamma$ the series

$$
\left(D_{x}^{\beta} D_{y}^{\gamma} \kappa_{z}\right)(x ; y)=\sum_{j=1}^{\infty} e^{-\lambda_{1} z}\left(D^{\beta} \varphi_{j}\right)(x) \overline{\left(D^{\gamma} \varphi_{j}\right)(y)}
$$

converges by the estimates (22) and that

$$
\left|\left(D_{x}^{\beta} D_{y}^{\gamma} \kappa_{z}\right)(x ; y)\right| \leqq c_{\beta, \gamma, t} e^{-\lambda_{1} t} e^{-b_{0}|x|^{1 / \alpha}-b_{0}|y|^{1 / \alpha}}
$$


with $t=\operatorname{Re} z$ and

$$
c_{\beta, \gamma, t}=c_{\beta} c_{\gamma} \sum_{j=1}^{\infty} e^{-\left(\lambda_{1}-\lambda_{1}\right) t} e^{2 k m n r j^{1 /(2 k n)}}<\infty .
$$

Note that $\sup _{t \geqq 1} c_{\beta, \gamma, t}<\infty$.

The foregoing estimates establish that the spectral decomposition (21) of the semigroup generated by $H$ is uniformly convergent. But as the estimates also give an exponentially decreasing bound it follows that the series is $L_{p}$-convergent for all $p$. This is a direct consequence of the Lebesgue dominated convergence theorem. Note that uniform convergence can also be deduced from cross-norm estimates on the semigroup by arguments similar to those on p. 247 of [Rob].

Acknowledgements. This work started while the second named author was visiting the Eindhoven University of Technology. He wishes to thank the EUT for financial support. The work was completed while the first named author was visiting the School of Mathematical Sciences at ANU. He wishes to thank the ANU for financial support.

\section{References}

[CoG] Corwin, L., Greenleaf, F.P.: Representations of nilpotent Lie groups and their applications Part 1: Basic theory and examples. Cambridge Studies in Advanced Mathematics 18, Cambridge etc.: Cambridge University Press, 1990

[Dav1] Davies, E.B.: One-parameter semigroups. London Math. Soc. Monographs 15, London etc.: Academic Press, 1980

[Dav2] Davies, E.B.: Heat kernels and spectral theory. Cambridge Tracts in Mathematics 92. Cambridge etc.: Cambridge University Press, 1989

[E1R] Elst, A.F.M. ter, Robinson, D.W.: Weighted strongly elliptic operators on Lie groups. J. Funct. Anal. 125, 548-603 (1994)

[GeS] Gel'fand, I.M., Shilov, G.E.: Generalized functions. Vol. 2, New York: Academic Press, 1968

[Gri] Grigor'yan, A.: Heat kernel upper bounds on a complete non-compact manifold. Rev. Mat. Heroamericana 10, 395-452 (1994)

[Kir] Kirillov, A.A.: Unitary representations of nilpotent Lie groups. Russian Math. Surveys 17, 53-104 (1962)

[KuS] Kusuoka, S., Stroock, D.: Long time estimates for the heat kernel associated with a uniformly subelliptic symmetric second order operator. Ann. Math. 127, 165-189 (1988)

[NSW] Nagel, A., Stein, E.M., Wainger, S.: Balls and metrics defined by vector fields I: Basic properties. Acta Math. 155, 103-147 (1985)

[Rob] Robinson, D.W.: Elliptic operators and Lie groups. Oxford Mathematical Monographs. Oxford etc.: Oxford University Press, 1991

[VSC] Varopoulos, N.T., Saloff-Coste, L., Coulhon, T.: Analysis and geometry on groups. Cambridge Tracts in Mathematics 100. Cambridge: Cambridge University Press, 1992 
